\title{
Video-based human behavior understanding: a survey
}

\author{
Paulo Vinicius Koerich Borges, Nicola Conci, Andrea Cavallaro
}

\begin{abstract}
Understanding human behaviors is a challenging problem in computer vision that has recently seen important advances. Human behavior understanding combines image and signal processing, feature extraction, machine learning and 3D geometry. Application scenarios range from surveillance to indexing and retrieval, from patient care to industrial safety and sports analysis. Given the broad set of techniques used in videobased behavior understanding and the fast progress in this area, in this paper we organize and survey the corresponding literature, define unambiguously key terms and discuss links among fundamental building blocks ranging from human detection to action and interaction recognition. The advantages and the drawbacks of the methods are critically discussed, providing a comprehensive coverage of key aspects of video-based human behavior understanding, available datasets for experimentation and comparisons, and important open research issues.
\end{abstract}

\section{INTRODUCTION}

The capability of automatically detecting people and understanding their behaviors is a key functionality of intelligent video systems. The interest in behavior understanding has dramatically increased in recent years, motivated by a societal needs that include security [1], natural interfaces [2], gaming [3], affective computing [4] and assisted living [5]. Significant technological advances in hardware and communication protocols are also facilitating new services such as real-time collection of statistics on group sports [6] and annotation of videos for event detection and retrieval [7]. A number of processing steps are necessary to analyze the scene at different levels of abstraction, starting from the behaviors of objects of interest. The first step consists in detecting and tracking subject(s) of interest to generate motion descriptions (e.g. motion trajectory or combination of local motions), which are then processed to identify actions or interactions. When considering local motions, the analysis generally deals with a fine-grained level of understanding to recognize gestures and motion patterns at intra-body level [8]. Depending on the quality of the camera view, position information can be complemented by other descriptors, such as the trajectories of body joints [9] or head pose changes [10].

Copyright (c) 2013 IEEE. Personal use of this material is permitted. However, permission to use this material for any other purposes must be obtained from the IEEE by sending an email to pubs-permissions@ieee.org.

Paulo Vinicius Koerich Borges is with the Autonomous System Laboratory, ICT Centre, CSIRO. Address: 1, Technology Court, Pullenvale, QLD, 4069, Australia, paulo.borges@csiro.au,vini@ieee.org.

Nicola Conci is with the Department of Information Engineering and Computer Science, University of Trento, Via Sommarive 5, Trento, 38123 , Italy, conci@disi.unitn.it.

Andrea Cavallaro is with the Centre for Intelligent Sensing, Queen Mary University of London, Mile End Road, London, UK, Andrea.cavallaro@eecs.qmul.ac.uk

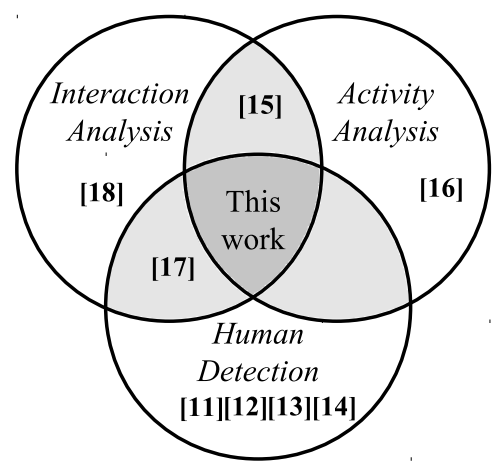

Fig. 1: A visual description of the contents of this work, in relation to previous surveys (indicated by the references).

Recognizing specific behaviors requires for example the definition of a set of templates that represent different classes of behaviors. However, in many scenarios not all behaviors can be characterized by a predefined number of classes nor can be known (and therefore represented) a priori. In such cases, it is common to use the concept of anomaly, namely a deviation from the learned behaviors [1]. In fact, although anomalous behaviors are generally difficult to model a priori, they can be detected as dissimilar from patterns acquired during regular behaviors.

Recent reviews on human behavior understanding focused on specific aspects of the overall problem, such as monocular pedestrian detection [11], human detection for driver-assistance systems [12] and pedestrian detection benchmarks [13], [14]. Morris and Trivedi [15] survey features and model motion trajectories focusing on trajectory analysis. Moeslund et al. [16] cover methods focusing on motion capture and analysis for human understanding. Comprehensive reviews on algorithms for detecting high-level behaviors compare activity recognition and interaction approaches [17]. Aggarwal and Ryoo [18] review action recognition approaches, extending actions and gestures to human-object interactions and group activities. However, the literature still lacks a comprehensive and up-to-date survey that discusses different abstraction levels at which behavior analysis can be developed depending on the application requirements and the granularity at which the captured information is represented. For this reason, in this paper we link fragmented aspects of this field in a coherent context for human behavior understanding from video (Figure 1). We present a critical analysis and a comparisons of relevant approaches, and we identify common features used by the methods and standard datasets to assess the performances of human behavior analysis algorithms. Moreover, because the 


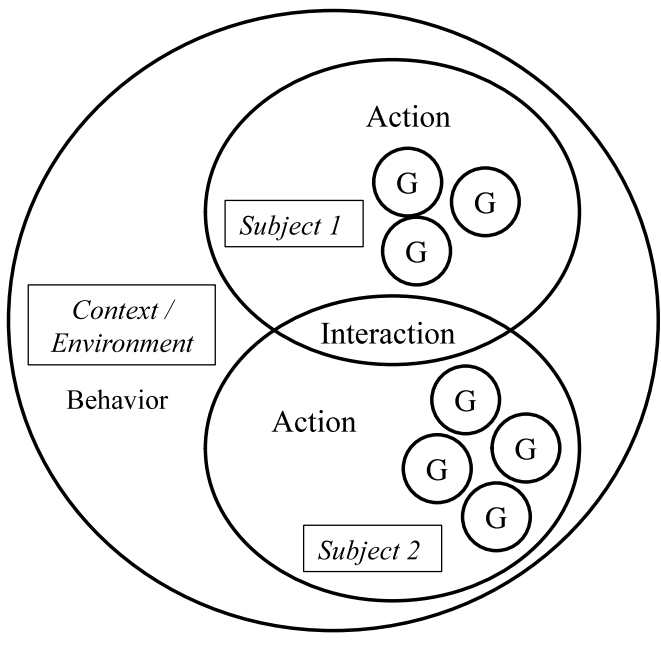

Fig. 2: A visual representation of the definitions for human behavior understanding as a combination of gestures (G), actions and interactions in a two-person scenario.

use of terminology is at times ambiguous in the literature, we provide a consistent definition of the terms used in human behavior analysis. Finally, we highlight open research challenges.

The paper is organized as follows. In Section II we introduce the definitions that will be used throughout the paper to describe human behaviors. Section III presents a review of human detection methods, whereas Section IV discusses actions and gestures. Next, Section V discusses how contextual information can be exploited to infer interactions. Finally, Section VI concludes the paper with a discussion and presents an overview on future trends and open research issues.

\section{DEFinitions}

Human activities can be categorized into four main groups, namely gestures, actions, behaviors, and interactions. Figure 2 illustrates the relationship between these groups in a twoperson scenario.

Gestures are movements of body parts that can be used to control and to manipulate, or to communicate [19]. Examples of gestures include stretching an arm and raising a leg. Gestures are the atomic components characterizing (describing) the motion of a person. From these atomic elements it is possible to compose actions, namely temporal concatenations of gestures [20]. Actions represent voluntary body movements of arbitrary complexity. An action implies a detailed sequence of elementary movements that make them univocally decodable by an observer and can be combined to compose single motion patterns or periodic motion patterns. Examples of single motion patterns are bending, jumping and grabbing an object; whereas examples of periodic motion patterns include walking, running and swimming.

A behavior is the response of a person to internal, external, conscious, or unconscious stimuli [21]. Unlike the recognition of actions that are represented by a sequence of characterizing visual elements, the recognition of behaviors requires a

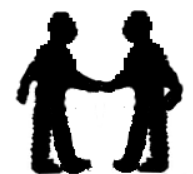

(a)

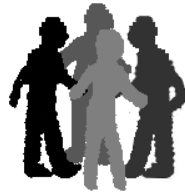

(b)
Fig. 4: Examples of (a) one-to-one interaction and (b) group interaction.

joint analysis of the (image) content and the (scene) context [22]. While actions can be analyzed in terms of motion and appearance features, the analysis of behaviors also requires information about the context and other factors influencing behaviors. Examples of contextual information include place and presence of other objects. Figure 3 illustrates how the same spatio-temporal features corresponding to the action of running lead to different behaviors depending on the context.

An interaction happens when other subjects or objects become a distinctive element to interpret the behavior of a person. We can distinguish between interactions with objects and social interactions. Interactions with objects happen when for example people use kitchen appliances, manipulate doors, or operate ATMs. Social interactions can be divided into twoperson and group interactions (Fig. 4). A social interaction implies a feedback mechanism that contributes to establishing a two-way communication [23], [24]. Examples of interactions are shaking hands and fighting, but also 'a person stealing a bag from another', since stealing serves as a stimulus to establish a relationship between the two people. Note that this definition differs from that by Turaga et al. [20] that made no distinctions between activities and interactions. Group interactions extend the concept of one-to-one interactions to multiple people sharing a common objective, such as 'a group marching' and 'a group meeting'. The common objective is in general regulated by a set of implicit or explicit rules, which are leveraged to alternate the action-reaction loop. As the number of people increases, it becomes more difficult to isolate them and to understand what they are doing, mainly due to occlusions and the difficulty in interpreting the role of each person in the group.

\section{Human Detection}

Video-based people detection methods can be divided into three main classes, namely appearance-based, motion-based and hybrid methods. Appearance-based techniques are a specific case of object detection in still images and use for example edge information. Motion-based methods consider temporal information for the definition of the features defining a human, in particular the movements of the legs. Hybrid methods use combinations of the previous two classes. Table I compares the characteristics of human detection methods, which will be discussed next together with available datasets to test their performance. 


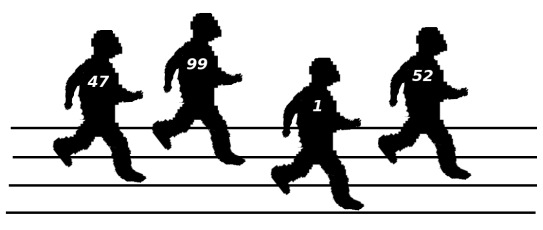

(a)

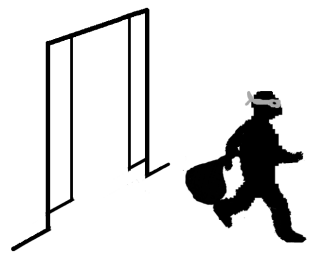

(b)

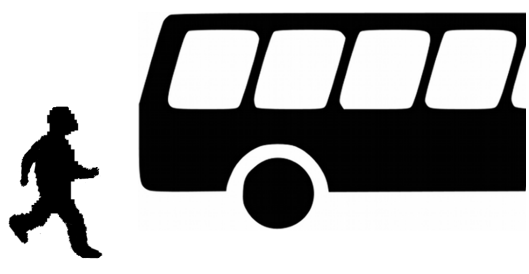

(c)

Fig. 3: Different behaviors generated by similar spatio-temporal features corresponding to the action of running: (a) sports; (b) robbery; (c) catching a bus.

TABLE I: Characteristics of human detection approaches (Key. DR: Detection Rate (\%); DT: Detection Time (fps); HOG: Histogram of Oriented Gradients; SVM: Support Vector Machine; MPL: Multi-Pose Learning).

\begin{tabular}{lll} 
Ref. & Features & Classifier \\
\hline$[25]$ & Appearance, HOG & linear and kernel SVM \\
{$[28]$} & Appearance, shape (local and global) & Probabilistic top-down segmentation \\
{$[26]$} & HOG and pictorial structures [29] & SVM \\
{$[27]$} & $\begin{array}{l}\text { Edge orientation histograms, a varia- } \\
\text { tion of HOG }\end{array}$ & SVM \\
{$[30]$} & Motion History Image & Metric thresholding \\
& & Spectral similarity comparison \\
{$[31]$} & Motion power spectral analysis & Boosting \\
{$[32]$} & Contour motion and Haar-like features & Metric based \\
& & cyclic pattern in human movement \\
{$[33]$} & Skeleton extremities & Linear Kernel SVM and MPL \\
{$[34]$} & Maximal principal gait angle & Boost [36] \\
{$[35]$} & Optical flow and HOG & Random Decision Forest \\
& & Kernel SVM \\
{$[37]$} & Motion point trajectories & Boosting \\
& & Linear SVM \\
{$[38]$} & Appearance, overall blob motion & Model fitting metric \\
{$[39]$} & Infrared HOG and Haar &
\end{tabular}

\section{A. Appearance-based methods}

Algorithms based on appearance features are specialized detectors trained on large pedestrian databases [12][11]. These algorithms generally scan each frame, searching for patterns that match pre-defined models. Appearance-based methods can be directly applied to non-static cameras (e.g., for automatic driver-assistance) [42] [43]. Effective representations include the histogram of oriented gradients (HOG) [25], the Viola and Jones algorithm [44] and Haar-like features [45].

Using HOG, the local shape of a human can be represented by the distribution of edge directions, given by intensity gradients. HOGs are usually combined with a support vector machine (SVM) classifier. However, despite being significantly invariant to scale and illumination transformations, HOGs change with object orientation. Several extensions of this representation have been proposed using the concept of HOGs for different body parts that are then combined [46] [29]. Another popular approach is the combination of local and global cues via a probabilistic top-down segmentation [28]. A combination of multiple features such as silhouette, appearance, holistic, and part-based can be used as input to

\begin{tabular}{|c|c|c|}
\hline DR & DT & Comments \\
\hline 89 & - & Landmark algorithm, extended in [26],[27] \\
\hline 71 & - & Good results for partially occluded humans \\
\hline 82 & - & Uses rendered synthetic pedestrian models for training \\
\hline 80 & 5 & $\begin{array}{l}\text { Part-based: lower dimensionality than traditional HOG; better perfor- } \\
\text { mance on INRIA dataset }\end{array}$ \\
\hline - & $\begin{array}{l}30- \\
50\end{array}$ & Uses motion characteristics of legs and vehicles \\
\hline 91 & 1.1 & Not only detection but also activity classification \\
\hline $90+$ & 24 & $\begin{array}{l}\text { Combined motion and static image analysis. Very good results on the } \\
\text { INRIA dataset }\end{array}$ \\
\hline 98 & 25 & Good results but skeleton analysis requires high quality segmentation \\
\hline 90 & 10 & Use phase-locked-loop \\
\hline $95+$ & - & Very good results on the Caltech and TUD-Brussels datasets \\
\hline- & $\begin{array}{l}2- \\
5\end{array}$ & Moving camera and near range \\
\hline $88+$ & 15 & Blob motion statistics combined with appearance methods \\
\hline 93 & 20 & Tuned HOG detector, optimized to the infrared domain \\
\hline 85 & 33 & Combination of $2 \mathrm{D}$ and $3 \mathrm{D}$ data, adapting HOG to RGB-D \\
\hline 98.4 & - & $\begin{array}{l}\text { 3D surface model for the head using RGB-D combined with } 2 \mathrm{D} \\
\text { contour model }\end{array}$ \\
\hline
\end{tabular}

a SVM classifier [27] [47]. The Viola and Jones algorithm, used initially in face detection, can be trained for pedestrian detection [48]. Haar-like features have also been used in combination with covariance features [45], leading to computationally efficient algorithms with performance comparable to HOGs in the INRIA dataset [49].

Benchmark papers on appearance-based people detection introduce a database with richly annotated videos and discuss evaluation measure for performance comparison [13], [14]. Pishchulin et al. [26] perform training on synthetically generated models, by employing a rendering-based reshaping technique to create thousands of images from only a small number of real images.

\section{B. Motion-based methods}

Motion-based methods generally detect the cyclic motion of the legs and assume a static camera to identify the moving foreground [50]. Although joint segmentation and detection processes have been proposed [28] [51], person detection often considers a blob segmented from the background, over which further analysis is performed to verify whether the blob is a 
pedestrian. However, when under-segmentation occurs, these methods become unreliable. An alternative is to analyze the motion statistics of the tracked blobs as a whole. Useful cues include the cyclic pattern in blob trajectory and an in-phase relationship between change in blob size and position [52].

When observing cyclic motion, gait is analyzed based on pixel or region-wise oscillations, such that the general statistical periodic behavior is used for classification. Early works performed a Discrete Fourier Transform (DFT) to quantify pixel oscillations [53] [54]. Variations of the method analyze the power spectral similarity in the walking pattern [31] or the amount of change in a motion history image [30]. An alternative to analyzing the full pixel intensity information is to high-pass filter the image, observing only a contour motion feature [32]. Cutler and Davis [55] look for the gait period by calculating a similarity matrix for every image pair in a sequence. He et al. [33] determine the angle formed by the centroid point and the two bottom end points of the object skeleton. The histogram of this angle over time is used for detection.

Other periodic detection algorithms use the Phase-Locked Loop [56] and autoregressive moving average models (ARMA) for estimating frequencies of sinusoidal signals. For example, Quinn and Hannan [57] use a second order ARMA model and derive theoretical performance bounds. Another well-known frequency estimation method is the multiple signal classification (MUSIC) algorithm, which estimates the frequency content of a signal using an eigenspace method [58]. Ran et al. [34] discuss the methods above addressing the specific problem of periodicity estimation for pedestrian detection. Other works analyzed the periodic change in the optical flow [35] [59]. Perbet et al. [37] use a number of point trajectories and identify those which are spatio-temporally correlated as arising from feet in walking motion.

People detection performance can be improved by considering scene modeling that helps reducing the search space.

Knowledge of the scene can be used to train for a specific area [60] [61]. The adaptation of a generic pedestrian detector to a specific traffic scene can be performed automatically [61], adding for example information about common pedestrian appearance in that scene, and the most likely paths. Moreover, when the homography between the ground and the camera is known, size features can be used for the detection [62]. Liu et al. [63] manually annotate the main surfaces in the scene providing spatial contextual knowledge. The density distribution over surfaces is learned from the scene and it is conditional on the surfaces semantic properties. As an example, on a zebra crossing pedestrians occur with a higher probability than cars, for a given direction of movement. All these cues improve the pedestrian detection reliability.

People detection in the infrared domain can be achieved with slight modifications to visible-spectrum detectors [39]. Moreover, infrared imaging presents particular contrast characteristics that can be exploited [64] [65]. Although simple brightness analysis can be inefficient for characterizing people due to the polarity switch phenomenon [65], employing appropriate statistical models such as the Infinite Gaussian Mixture Model [64] for the contrast around potential areas yields effective classification.

\section{Hybrid methods}

Efficient people detection solutions combine appearance and motion [48], [66]. These two approaches can separately analyze the data, merging the final result according to a given decision function, or apply a still-image detector to regions potentially containing pedestrians, as indicated by a blob tracker. The advantage of the former solution is that the detection is not restricted to moving blobs. However, the computational complexity is largely increased, as the appearance-based detector performs a search over the full frame, reducing the number of frames per second (fps) in video analysis. The latter solution reduces computational complexity and the false-positive rate.

Methods that consider a background model to segment potential pedestrians generally present a better detection rate, both in terms of false positives and false negatives.

One of the earlier works jointly exploiting both types of features combines a Viola and Jones detector [44] operating on differences between pairs of images [48] and training using AdaBoost [67]. Each step of the AdaBoost selects from the several motion and appearance features the feature with lowest weighted error on the training samples. The final classifier weighs pixel intensity and motion information such that the detection rates are maximized. The accuracy of this technique [48] can be improved by one order of magnitude at the expense of using a higher number of frames [68]. A related approach is also used by Dalal [66] and Bouchirika et al [69], combining HOGs with motion and gait patterns. The concept can be extended to also include behavior as a cue in the detection process [70], where blobs with affine movements have a higher likelihood of representing a group of pedestrians.

Alternative sensing modalities using depth information are becoming increasingly popular. Xia et al. [41] perform detection by using a 2-D head contour model and a 3-D head surface model, in a constrained office area. An adaptation of HOGs to RGB-D data is proposed by Spinello and Arras [40], where the direction of depth changes are locally encoded. An accuracy of $85 \%$ in ranges up to 8 meters is achieved.

\section{Datasets}

Datasets for testing human detection algorithms include the MIT Pedestrian Database [71], the USC Pedestrian Detection Testsets [72], [73], the CALTECH Database [13], the INRIA Person Dataset [49] and the ETH Dataset [74]. The MIT Pedestrian Database was one of the earliest datasets, with the people poses limited to frontal and rear views. The images are scaled to the size $64 \times 128$ and aligned such that the person's body is centered in the image. The INRIA Person Dataset presents similar scenarios but with a larger variety in poses and comprehensive annotation. Later datasets [72], [13] contain lower resolution pedestrians in larger images, recorded in cluttered urban areas. These datasets contain rich annotation, including occlusion information.

The PETS Database [75] contains single and multi-view footages including outdoor and indoor scenarios. The ETH 
A
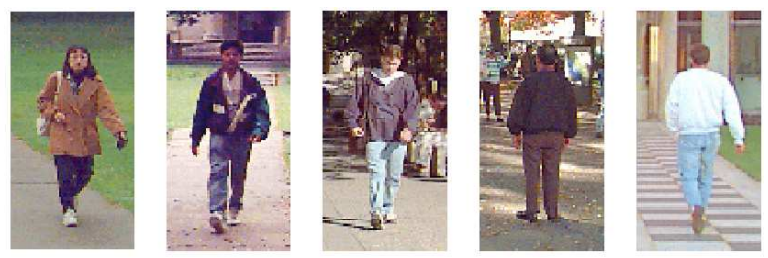

B
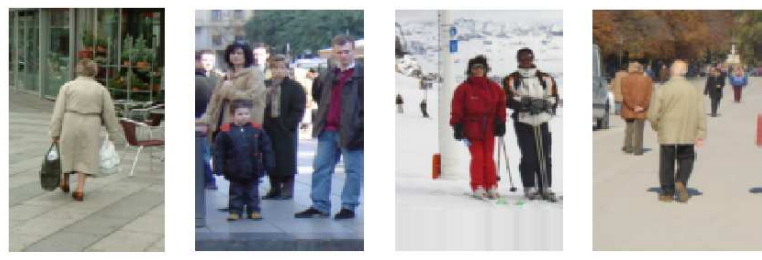

C
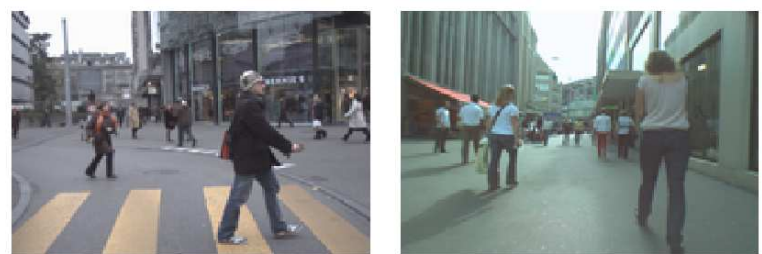

Fig. 5: Samples of different datasets used for training and evaluation of human detection algorithms. Scenarios considered range from simple cropped images (row A) to wide views with multiple people and complex background (row C). Dataset Key: A: MIT [71]; B: INRIA [49]; C: ETH [74].

Dataset [74] is collected using two cameras, with annotation and camera calibration information. A comprehensive discussion on datasets for pedestrian detection is presented in [14].

Sample images from detection datasets are shown in Figure 5, illustrating from top to bottom how the complexity of the scenarios has evolved over time.

\section{ACTIONS}

Action recognition approaches commonly use as descriptors low-level features such as optical flow (both dense and sparse) with gradient information [77], [78], silhouettes [79], and longterm analysis of motion [80]. Semantics and context-based techniques can be used to support and increase the system reliability [81], [82]. A selection of relevant techniques for action recognition using these techniques are described below and compared in Table III.

\section{A. Low-level features and spatio-temporal interest points}

Common approaches for action recognition include dense optical optical flow [95], [97] and spatio-temporal interest points (STIPs) [86], [78], [98], [99], [100], [101], [102]. Laptev and Lindeberg originally presented STIPs for action recognition [103] as a space-time variation of the Harris corner detector [104]. Regions with high intensity changes in both space and time are defined as STIPs. Alternatively temporal Gabor filters can be employed to increase the robustness of the method [98], [105]. STIPs do not necessarily rely on a person detector, they are robust to clutter and are relatively invariant to temporal and appearance changes. However, they are generally combined with bag-of-features models and therefore do not account for temporal and spatial probabilistic feature distributions.

Early work by Schuldt et al. [78] considered individual feature detections to be independent and built a single histogram for the full video sequence. Ullah et al. [106] perform video segmentation prior to STIP detection to eliminate misplaced interest points. Alternative approaches use several binning designs [107], [108] to obtain the relative structure of STIPs across a video. Although results are generally better than the single-bin (histogram) approach, rigid bin partitions can affect the spatial or temporal shift in the video volume. Chakraborty et al. [109] introduce a bag of visual words vocabulary approach by combining spatial pyramid and vocabulary compression techniques.

Dense optical flow has also been successfully used for action recognition [95], [97], [110]. Wang et al. [95], [110] use dense optical flow trajectories and segment the foreground from background motion for moving cameras. Dense trajectories are more robust to irregular abrupt motions and are more accurate in capturing complex motion patterns. The authors show that motion boundaries encoded along the trajectories can outperform state-of-the-art trajectory descriptors, on the KTH [78], Youtube [111], Hollywood2 [112] and UCF Sport datasets [113].

An action spectrogram for interest points in $3 \mathrm{D}$ volumes is used by Chen and Aggarwal [114], with successful results on the Weizmann dataset and $91 \%$ accuracy on the KTH dataset. A variation of the problem is to consider not only action recognition, but also action prediction [115], with $70 \%$ accuracy in UT Dataset [116].

Given the redundancy of information collected through spatio-temporal features, Castrodad and Sapiro [96] propose a sparse coding pipeline, by classifying the incoming videos using a dictionary of learned primitives. In spite of the simplicity of the method, the achieved results reach very high performances, scoring $100 \%$ on the KTH and UT datasets, and $96 \%$ on the UCF-Sports dataset.

\section{B. Mid and high-level representation}

At a higher level, action recognition can be performed by exploiting mid and high-level features, such as long-term tracked trajectories and semantics. Choi et al. [80] present a framework for collective activity recognition exploiting motion trajectories and poses. By analyzing coherent activity, better action recognition performance is obtained. Using their own dataset [117] with actions such as standing, queuing, walking and talking, they obtain an average accuracy of $82 \%$. Raptis et al. [92] use motion trajectories to spatially locate where an event occurs. Trajectories are clustered, and the clusters' properties are analyzed to classify the action. They achieve a $79.4 \%$ accuracy on the UCF-Sports Dataset. Hoai et al. [93] present a framework for joint segmentation and recognition of actions based on multi-class SVM for action classification, while segmentation inference is achieved by dynamic programming. Regular expressions can be exploited to automatically associate an observed activity pattern to a template of activities learned a priori, achieving a $87.7 \%$ accuracy on 
TABLE II: Datasets for human detection. (Key. A: Annotation; Y: yes; N: no; P: partial)

\begin{tabular}{|c|c|c|c|c|}
\hline Ref. & Name & Features & $\mathbf{A}$ & Comments \\
\hline [71] & MIT Pedestrian Database & 923 images, with windows around the subjects & $\mathrm{N}$ & Pose of people limited to frontal and rear views. Images scaled to $64 \times 128$ \\
\hline $\begin{array}{l}{[73],} \\
{[72]}\end{array}$ & $\begin{array}{l}\text { USC Pedestrian Detec- } \\
\text { tion Testset }\end{array}$ & 369 images, with 816 humans & $\mathrm{Y}$ & $\begin{array}{l}\text { Images collected from the Internet and from the CAVIAR video database [76], } \\
\text { with frontal/rear view walking/standing human }\end{array}$ \\
\hline [49] & INRIA Dataset & Approx. 2500 images, $128 \times 94$ & $\mathrm{Y}$ & $\begin{array}{l}\text { Refined combination of images from different datasets (including Internet), with } \\
\text { pedestrians with bounding box area }>100 \text { pixels }\end{array}$ \\
\hline [74] & ETH Dataset & 12.298 pedestrians in approx. 2.000 frames & $\mathrm{Y}$ & $\begin{array}{l}\text { Two-camera dataset. The unbayered images, the camera calibration, and annotation } \\
\text { are provided for both cameras. Annotated pedestrians are taller than } 50 \text { pixels }\end{array}$ \\
\hline $\begin{array}{l}{[13],} \\
{[14]}\end{array}$ & $\begin{array}{l}\text { Caltech Pedestrian De- } \\
\text { tection Benchmark }\end{array}$ & 10 hours of $640 \times 480$ video & $\mathrm{Y}$ & $\begin{array}{l}\text { Recorded from moving vehicle in urban area. } 350,000 \text { bounding boxes with } 2300 \\
\text { unique pedestrians annotated }\end{array}$ \\
\hline
\end{tabular}

TABLE III: Comparative analysis of action recognition algorithms (Key. Ref: Reference; DR: Detection Rate; MEI: MotionEnergy Image; MHI: Motion-History Image; GMM: Gaussian Mixture Model; HMM: Hidden Markov Model; HMM-MIO: HMM with Multiple Independent Observations; SVM: Support Vector Machine: EM: Expectation Maximization; MRF: Markov Random Field)

\begin{tabular}{|c|c|c|c|}
\hline Ref. & Representation & Classifier & Key Aspects \\
\hline [83] & MEI and MHI & "Pooled" Mahalanobis distance & Temporal templates and dynamic matching. Real time and multi-view \\
\hline [84] & Fourier Descriptors & SVM and HMM & $90 \%$ DR on ad-hoc dataset with 101 activities (5 subjects) \\
\hline [85] & GMM to model salient postures & EM-based learning & Tolerance to noise, robustness across subjects and datasets \\
\hline$[86]$ & Spatio-temporal template (star-figure) & GMM framework & $\begin{array}{l}\text { GMM features arranged in a two dimensional map, transformed into a gray } \\
\text { level image }\end{array}$ \\
\hline [87] & Hierarchical & $\chi^{2}$ test & $\begin{array}{l}\text { Automatic model adaptation; unsupervised behavior analysis and abnormality } \\
\text { detection }\end{array}$ \\
\hline [88] & Action graphs and 3D points & Bi-gram maximum likelihood decoding & $\begin{array}{l}\text { Sparse sampling of } 3 \mathrm{D} \text { points to characterize the } 3 \mathrm{D} \text { shape of each salient } \\
\text { postures }\end{array}$ \\
\hline [89] & $\begin{array}{l}\text { HMM with multiple independent obser- } \\
\text { vations (HMM-MIO) }\end{array}$ & HMM & $\begin{array}{l}\text { Robustness to outliers, dimensionality reduction, handles sparse observations; } \\
\text { performance comparable to discriminative classifiers }\end{array}$ \\
\hline [90] & Self similarity matrix & Nearest neighbor & $\begin{array}{l}\text { Self similarity matrices combined over time. } 100 \% \text { accuracy on KTH and } \\
\text { Weizmann datasets }\end{array}$ \\
\hline [91] & Motion trajectory & $\begin{array}{l}\text { SCFG, Allen's temporal logic, Multi- } \\
\text { Thread Parsing }\end{array}$ & Error detection and recovery, automatic rules induction \\
\hline [80] & $3 \mathrm{D}$ trajectories and pose & Random Forest and 3D-MRF & Group analysis as the spatial distribution of people and its temporal evolution \\
\hline [92] & Motion trajectories & SVM, MRF & Classification based on trajectory grouping \\
\hline [93] & Low or mid-level features & SVM, Dynamic Programming & Joint segmentation and classification of actions \\
\hline [82] & Template-based action detector & SVM & High-level representation. Invariance to scale, viewpoint and tempo \\
\hline [94] & Motion trajectories & Spectral analysis of graphs & Trajectories as transitions of graph nodes \\
\hline [95] & Dense optical flow & Motion boundary histogram & $\begin{array}{l}\text { Dense optical flow trajectories and an efficient tracker and descriptor, seg- } \\
\text { menting foreground motion from background motion }\end{array}$ \\
\hline [96] & 3D image patches & Sparse coding & $\begin{array}{l}\text { Dictionary learning to extract action primitives and used as basis vectors. } \\
\text { Classification obtained by reconstruction }\end{array}$ \\
\hline
\end{tabular}

the Weizmann dataset and $42.4 \%$ on the Hollywood dataset. Daldoss et al. [118] model actions through an abstraction of the top-view trajectory. The expressions corresponding to the activity models are learned as separate Context-Free Grammars (CFGs) using a set of training sequences, parsed using the Earley-Stolcke algorithm. Zhang et al. [91] transform the motion trajectories in a set of basic motion patterns and use a rule induction algorithm based on the Minimum Description Length (MDL) to derive the spatio-temporal structure of the event from the primitives stream. Anomalous actions can be detected based on spectral analysis of graphs [94] by representing human motion trajectories as transitions between nodes. Individuals span only a limited portion of all possible trajectories on the graph and this subspace is characterized by large connected components of the graph, on which it is possible to implement invariant metrics for anomaly detection.

Most of the works discussed above define human actions by their motion and appearance features. However, other contex- tual and semantic information can also be employed [119], [81], [82]. Gupta et al. [119], use a storyline to describe causal relationships between actions (e.g. baseball swinging and running are typically related actions). AND-OR graphs are used as a mechanism for representing storyline models. The parameters and structure of the graph are learned from weakly labeled videos, using linguistic annotations and visual data, leading to joint learning of both storyline and appearance models. Lin et al. [81] use attribute graphs for activity recognition. They model the variability of semantic events by a set "event primitives", which are learned as a object-trajectory mapping that describes mobile object attributes (location, velocity, and visibility). With this representation, one observed event is parsed into an event parse graph, and variabilities of one event are modeled into an AND-OR graph.

Raptis and Segal [120] model an action as a very sparse sequence of temporally local discriminative keyframes. Keyframes are learned as collection of poselets, a description 
recently used in person recognition [121]. Poselets capture discriminative action parts that carry partial pose information, thus reducing the impact of occlusions. The model semantically summarizes actions in a storyline, which represents a contextual temporal orderings of discriminant partial poses.

Inspired by the object bank approach for image segmentation [122], Sadanand and Corso [82] propose a high-level representation of actions. The main idea is that a large set of action detectors can be seen as the bases of a high-dimensional "action-space". This characteristic, combined with a simple linear classifier, can form the basis of a semantically-rich representation for activity recognition in video. The authors report accuracy of $98.2 \%$ on the KTH, $95.0 \%$ on the UCF Sports, and 57.9\% on the UCF50 dataset. Promising results are also given by Messing [123], where the semantics are incorporated to his previous work [124] on activity recognition using a model for the velocity history of tracked points. Higher level information, the relative position of a person, their body parts and objects in known scenarios are incorporated in the activity recognition system. As the knowledge of objects aid activity recognition, Fathi et al. [125] use an egocentric approach, where video captured from a wearable camera is used to view the scene in front of the user at all times. They perform the analysis of hand movements and interaction with objects, using a weakly supervised technique. The method automatically segments the active object areas, assigning regions to each object and propagating their information using semisupervised learning.

\section{Silhouettes}

The use of silhouettes to classify actions assumes that human movements can be represented as a continuous progression of the body posture. These approaches are mainly based on background segmentation [11]. Action descriptors can be extracted from a sequence of silhouettes in consecutive frames and traditional classifiers can be employed for recognition [83], [126]. Alternatively a dynamic model of the action of interest can be generated using characteristics from each silhouette [79], [127].

Action descriptors extracted from a sequence of silhouettes capture and combine spatio-temporal characteristics of the activity. A common technique is to accumulate silhouettes to generate motion energy images (MEI) as well as motion history images (MHI) [83]. Hu moments [126] can be extracted from both MEI and MHI as action descriptors, and action classification is based on the Mahalanobis distance between each moment descriptor of the known actions and the one under analysis. Chen et al. [86] fit star figure models to human silhouettes to identify the five extremities of the shape corresponding to head, arms and legs. To model the spatial distribution of the five points over time, Gaussian Mixture Models are used, ignoring the temporal order of the silhouettes in the action sequence. Nater et al. [87] use a hierarchical approach based on silhouettes to detect falls, whereas Li et al. [88] extend the silhouette approach to 3D sensors.

Dynamic models of actions generated by extracting characteristics from each silhouette generally employ statistics-based techniques such as Conditional Random Fields (CRF) [128], [129] and Hidden Markov Models (HMM) [127], [89]. The features extracted from each silhouette capture the shape of the body and possible local motion. Kellokumpu et al. [84] apply Fourier-based shape descriptors to cluster a number of possible postures. HMMs are employed to model the activity dynamics, such that each cluster is assumed as a discrete symbol from the hidden states in the HMM. Instead of analyzing the behaviour of whole bodies, an alternative is to perform parts-based analysis [130], [131], where template matching is combined with a pictorial structure model to detect and localize actions.

Sun et al. [90] were the first to obtain 100\% accuracy on both the KTH and Weizmann datasets by generating a selfsimilarity matrix (SSM) for each frame in the video using a feature vector. The SSMs from all frames are combined and the result is decomposed into its rank-1 approximation, yielding a set of compact vectors that efficiently discriminate different actions. Alternative methods analyze skeletons [132], [85], [20], [16], which represent the human body in terms of articulated joints, thus extending the information provided by silhouette and shape to analyze motion and pose at a finer level of detail. In a simple form, skeleton-based articulation considers a small number of points: the head, hands, and feet, for example, forming a 5 point star [132]. Most statistics are derived from the absolute and relative motion of those points with respect to the star centroid. The statistics can be analysed directly or can serve as input to a classifier.

When subjects are close to the camera, using RGB-D, Shotton et al. [133] predict the 3D positions of skeletal body joints from a single image. Using a highly diversified training dataset, they generate an intermediate body parts representation that maps the complex pose estimation problem into a simpler per-pixel classification task. Extending the poses to activities, Sung et al. [134], [135] present learning algorithms to infer the activities, using a hierarchical maximum entropy Markov model on RGB-D data. Their method considers an activity as composed of a set of sub-activities of body parts, inferring a two-layered graph structure using dynamic programming. They achieve an average precision rate of $84.3 \%$ in house and office environments.

\section{Datasets}

Activity recognition datasets are either staged or natural, and contain images or trajectory data. The KTH dataset [78] and the Weizmann dataset [136] contain instances of walking, jogging, running, boxing, hand waving, and hand clapping, performed several times by different subjects. State-of-the-art methods achieve recognitions rates of more than 90\% [131], [137] in the KTH and Weizmann datasets, which provide an initial basis for comparison among algorithms [89]. More realistic datasets include UCF50 [138] and Hollywood [137]. Other staged databases include INRIA Xmas [139] and MuHAVi [140], which contain multi-camera views. The UCF YouTube database [111] has large variations in camera motion, object appearance and illumination conditions, as illustrated in Figure 6. Large variations are also found in the Hollywood Human Action datasets [137], [112], which contain thousands 
A

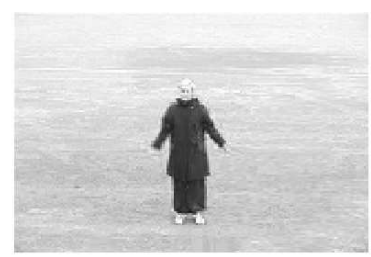

B

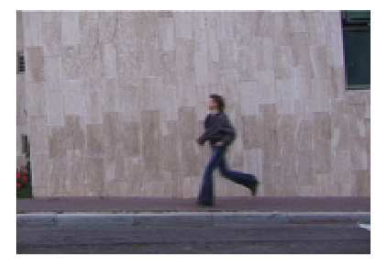

C

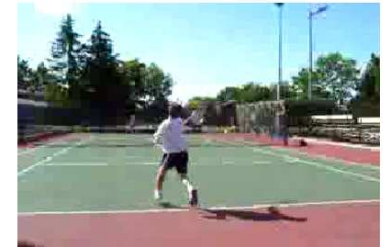

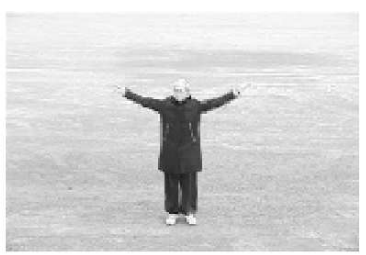
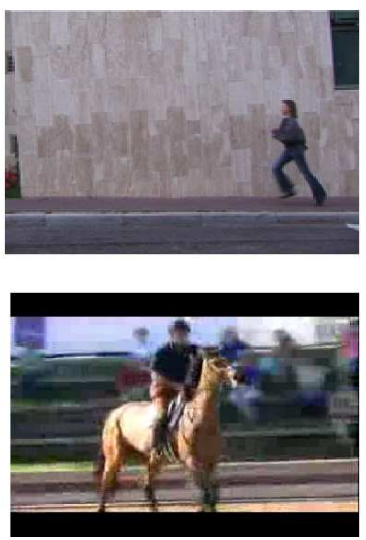

Fig. 6: Samples of different datasets used for training and evaluation of human activity classification algorithms. The examples include staged scenarios (rows A and B) and real sequences obtained from the Internet (row C). Dataset Key: A: KTH [78]; B: WEIZMANN [136]; C: YouTube [111].

of video movies clips corresponding to more than 20 hours of data representing 12 human actions classes and 10 scene classes (e.g. kitchen, car, hotel).

Other datasets only consider the spatio-temporal motion information provided by the trajectories of moving people. These datasets include the 3D People Surveillance Dataset 3DPES [141], the MIT trajectory dataset [142] and the Edinburgh Informatics Forum Pedestrian Database (EIFPD) [143]. The 3D People Surveillance Dataset 3DPES [141] contains paths of about 200 people, acquired over several days and complemented with ground-truth information in the form of bounding boxes and direction of motion. The MIT trajectory dataset [142] contains more than 40,000 trajectories collected over five days from a single camera monitoring a parking lot. The Edinburgh Informatics Forum Pedestrian Database (EIFPD) [143] covers several months of observation resulting in about 1000 trajectories/day. Although data do not include the ground truth, information about the detected blobs, the raw samples, and the interpolated trajectory using splines is provided.

As subset of activity recognition, datasets for gesture recognition include ASL (Australian Sign Language) [144], FGnet [146], [149], [147], Pointing'04 [148], Cambridge Gesture Database [150], and the ChaLearn Gesture Challenge dataset [151]. The ASL [144] dataset consists of a wide set of samples of Auslan (Australian Sign Language) signs: 27 examples of each of 95 Auslan signs were captured from a native signer using high-quality position trackers. A small two-handed dataset is presented in [146] provided by the Face and Gesture Recognition Working Group (FGnet). The videos contain 7 two-handed gestures, collected using 7 different

subjects and recording 10 videos per gesture per subject. The same working group proposed in [145] additional datasets for hand posture recognition, as well as a specific dataset for hand gesture recognition [147]. This dataset includes deictic and symbolic gestures for 400 samples. The benchmark data of Pointing'04 workshop [148] include ground-truth information indicating where users are pointing. Nine static and four dynamic gestures are presented and annotated in [149], including besides the identifiers for event and user, the gesture, action start, stroke start, stroke end and gesture end. 900 image sequences of 9 gesture classes defined by 3 primitive hand shapes and 3 primitive motions are presented in [150]. Each class contains 100 image sequences (5 different illuminations, 10 arbitrary motions, 2 subjects). The target task for the data set is to classify different shapes as well as different motions at a time.

Recent RGB-D datasets include the RGBD-HuDaAct [152] and the ChaLearn Gesture Challenge [151] datasets. The RGBD-HuDaAct database contains videos showing subjects performing various common daily life activities, such as talking on the phone, getting up, and mopping the floor. The dataset is annotated and one single subject is present in each action. Each sequence spans about 30 to 50 seconds, totaling approximately 46 hours of video at $640 \times 480$ resolution. Each subject performs the same activity 2-4 times. In the ChaLearn Gesture Challenge dataset [151] ground-truth annotation is partially available, including temporal segmentation into isolated gestures and body part annotations (head, shoulders, elbows, and hands).

A summary of the characteristics of the datasets discussed above is given in Table IV.

\section{INTERACTIONS}

We can distinguish between one-to-one and group interactions. One-to-one interactions can be seen as extensions of action for single subjects complemented by contextual and social information. Group interactions require the detection of a group entity in terms of social aggregation. In both cases motion features need to be combined with sociological and psychological information that rule interpersonal relations. However, the literature usually addresses them as two separate problems. Table $\mathrm{V}$ compares the interaction recognition techniques described below.

Recognizing the social component of behaviors and social activities that involve multiple people requires observing the activity of a person in reference of his/her neighbors. Considering position as the main source of information, interactions can be modeled by the permanence for a specific amount of time in the neighborhood of one or more meaningful spots in the scene. Models from psychology and sociology that can be used include the theory on proxemics [153][154]. Proxemics exploits the so-called social space (the space between subjects) in order to infer interpersonal relationships. These relationships can be seen as a summation of attractive and repulsive forces that drive human behaviors, linking them with other people in the surroundings. Helbing et al. [155] assimilate the human behavior in a social context as a summation of forces that 
TABLE IV: Datasets for gesture recognition. (Key. A: Annotation; Y: yes; N: no; P: partial; CGD: Cambridge Gesture Database; CGC: ChaLearn Gesture Challenge)

\begin{tabular}{|c|c|c|}
\hline Ref. & Name & Features \\
\hline [144] & ASL & 95 signs, 27 examples per sign \\
\hline [145] & FGNet & $\begin{array}{l}10 \text { postures, } 24 \text { people, } 3 \text { backgrounds for static; } 1 \text { sequence, } \\
4 \text { gestures for dynamic }\end{array}$ \\
\hline [146] & FGNet & 7 gestures, 7 users, 10 videos per gesture \\
\hline [147] & FGNet & about 400 videos \\
\hline [148] & FGNet & 8 videos, each with user pointing in 8 directions \\
\hline [149] & FGNet & 13 gestures, 9 static, 4 dynamic, 16 users \\
\hline [150] & CGD & $\begin{array}{l}900 \text { sequences, } 9 \text { gestures, defined by } 3 \text { primitive hand } \\
\text { shapes and } 3 \text { primitive motions. }\end{array}$ \\
\hline [151] & CGC & about 50.000 images \\
\hline
\end{tabular}

lead a subject to its target (Social Force Model). The idea of a social force that binds and regulates the relationships between subjects has been widely adopted to model both one-to-one interactions and group interactions, as a generalization of the one-to-one case. Mehran et al. [156] use grid of particles over the image that are moved by the forces created by the spacetime optical flow as they were individuals. The moving forces are then estimated using the social force model to identify normal or abnormal behaviors.

\section{A. One-to-one interactions}

The interaction level between two subjects can be measured as an energy function (or potential) computed along the axis connecting them [157]. Pellegrini et al. [158] applied this model to crowded scenes, considering single moving entities as agents. The motion of each agent is driven by its destination, and planned so as to prevent collisions with other moving objects. Every agent is associated with a set of features, comprising position, speed and direction of motion. Path planning is achieved by implementing a social force model to evaluate the interaction level between two subjects. From the analysis of the energy function, the authors propose a Linear Trajectory Avoidance (LTA) model, based on a short time prediction, to model the avoidance path. Longer predictions would lose effectiveness, because of the casualness of the human movement. Taj and Cavallaro [159] describe the coupling between multiple object states using Coupled HMMs on relative spatio-temporal features among the people under analysis. Rota et al. [160] recognize normal and abnormal two-people interactions exploiting proxemics cues combined with motion information, also revealing the intentionality of a specific interaction. Zen et al. [161] identify proxemics cues in a close-range analysis to discriminate personality traits such as neuroticism and extraversion, and use the collected data to construct a correspondent behavioral model. The social interaction cues are then used to complement a people tracker to improve its accuracy.
Comments

Samples of Auslan (Australian Sign Language) signs captured from a native signer

Static and dynamic hand posture database

Two-handed gestures

Hand posture recognition, including deictic and symbolic gestures

Includes a video set for pointing on a whiteboard in a multi-camera setup and a set of videos recorded using a head-mounted camera.

Y Static and dynamic gestures performed under different illumination conditions by different subjects.

$\mathrm{Y}$ Sequences recorded in front of a fixed camera having roughly isolated gestures in space and time. Videos are uniformly resized into $20 \times 20 \times 20$

$\mathrm{P} \quad$ The dataset includes high quality RGB videos complemented by depth information of single users.

\section{B. Group interactions}

Extending the concept to a group of several subjects implies analyzing the scene from the social viewpoint and the dynamics ruling these interactions are significantly different. Cristani et al. [162] detect the so-called F-Formations in the scene to infer whether an interaction between two or more persons is occurring. The interactions in a small groups of people can be encoded in three categories, namely self-causality, paircausality and group-causality [163], where features characterizing each category are identified based on the trajectories of the subjects.

The social interaction model proposed in [158] [164] assumes that people behave consistently when walking in groups. Trajectory hypotheses are generated for each person within a time window, and the best hypothesis is selected taking into account social factors, while estimating group membership using Conditional Random Fields. The group membership allows optimizing motion prediction also considering constraints imposed by the group. The algorithm improves tracking performance by introducing additional constraints for the tracker. Finally, an interaction energy potential can be extracted to model the relationships among groups of people [165]. The relationship between the current state of the subject and the corresponding reaction is then used to model normal and abnormal behaviors.

RGB-D data can be used to detect fine-grained humanobject interaction [166], identifying hand movements and other cooking activities, considering the interactions between hands and objects. The system combines global and local features, also considering the duration of the events. The global feature uses the principal component analysis (PCA) on the gradients of 3D hand trajectories. The local feature employs bag-ofwords of trajectory gradients snippets, which are useful for distinctive isolated actions such as chopping.

Zhang and Parker [167] employ RGB-D sensors on a mobile robotic platform, aiming at efficient interaction between the robot and humans. The feature detector applies separated filters on the 3D spatial dimensions and to the temporal dimension to detect a feature point. A feature descriptor then clusters the intensity and depth gradients within a 4D cuboid, which is 
centered at the detected feature point as a feature. As a classifier for the activity recognition, the authors employ the Latent Dirichlet Allocation [168] combined with Gibbs sampling. Detection rates are relatively high in a staged scenario, for basic activities such as waving, walking, and signaling. Koppula et al. [169] use a mobile robot and consider the problem of labeling sub-activities performed by a human, but also use interaction with objects as cues (associated affordances). The human activities and object affordances are jointly modeled in a Markov Random Field (MRF) framework. The learning problem is formulated using a structural SVM approach, with $75.8 \%$ precision and $74.2 \%$ recall in a challenging dataset.

\section{Datasets}

Interaction detection datasets include the BEHAVE dataset [170], the UT Interaction Dataset [116], the TV Human Interactions Dataset [171] and the CMU MOCAP [172] (Figure 7). The BEHAVE dataset [170] covers interactions between multiple persons with subtly different behaviors such as InGroup (IG), Approach (A), WalkTogether (WT), Split (S), Ignore (I), Following (FO), Chase (C), Fight (FI), RunTogether (RT) and Meet (M). A smaller dataset is the UT Interaction Dataset for the SDHA Contest [116] that covers continuous executions of 6 classes of human-human interactions: shakehands, point, hug, push, kick and punch. Ground-truth labels for these interactions are provided, including time intervals and bounding boxes. There are 20 video sequences of around 1-minute length. For close-range interactions, the TV Human Interactions Dataset (TV HID) [171] is used to analyze interactions occurring in TV footages [173]. The dataset is annotated. Finally, the CMU MOCAP [172] is an annotated 3D dataset that also includes a section for two-person interactions. The position and motion information of body parts are captured using markers that create the skeleton of the person. The dataset includes several types of behaviors, ranging from normal interactions such as handshaking and passing an object to quarrels and fights.

In the RGB-D domain, datasets include the Cornell CAD60 [134] and CAD-120 [169], and the LIRIS [174]. The LIRIS dataset contains video of multiple subjects, with partial focus on interactions, such as discussing, handling items, handshaking, apart from some single subject actions present in the RGBD-HuDaAct dataset. Full annotation is provided, with information on the type of action and related spatial and temporal position in the video. This dataset contains fully annotated standard $720 \times 576$ video of the same scenes, recorded with a consumer camcorder, providing a useful basis for comparisons between RGB and RGB-D techniques. The CAD-60 and CAD-120 datasets consider most environments of a regular household (office, kitchen, bedroom, bathroom), with three to four common activities identified in each location with about 45 seconds of data for each activity and each person. The CAD-120 extends the CAD-60 by separating high-level human-object interactions (e.g. taking medicine, cleaning objects, microwaving food) and sub-activities like punching, reaching, and drinking. Object characteristics labels such as movable, reachable and drinkable are also included.
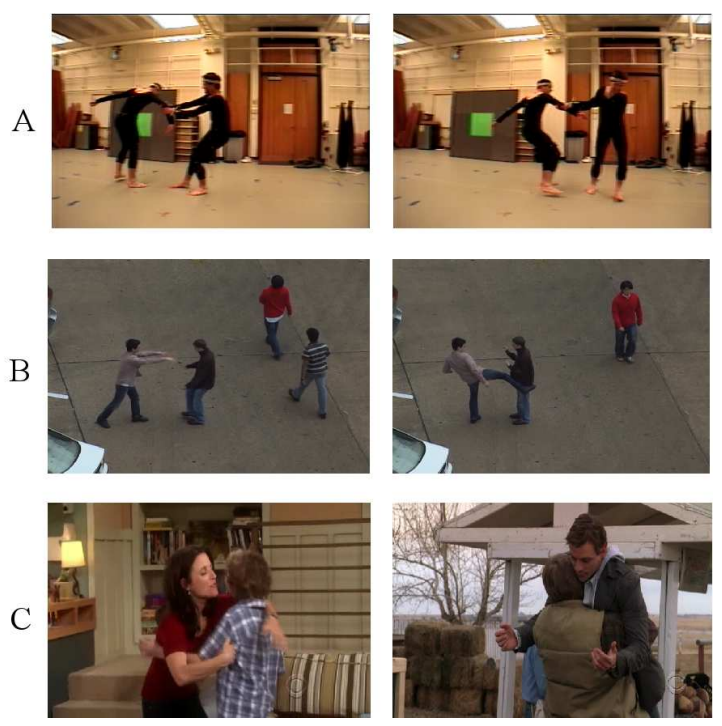

Fig. 7: Samples from different datasets used for training and evaluation of interaction classification algorithms. The examples include "pulling", "fighting" and "hugging". Dataset Key: A: MOCAP [172]; B: UT [116]; C: TV HID [173].

This dataset contains a comprehensive annotation and each video is annotated with sub-activity labels, tracked human skeleton joints and tracked object bounding boxes. This dataset combines actions, gestures and interactions in its annotations.

A summary of the characteristics of the datasets discussed above is given in Table VI.

\section{Discussion And Conclusions}

This survey presented an extensive overview of video-based behavior understanding, its related definitions and an analysis of datasets used for benchmarking. The goal is to provide the reader with a critical analysis of the major steps, from detection to high-level interpretation, contrasting key elements of the different approaches using comparative tables. Based on this survey, we can highlight successful approaches as well as several important future directions, which include promising methods, the generation and reconstruction of 3D observations, datasets and annotation.

From a representation viewpoint, and in spite of their simplicity, low-level features (such as pixels), but also spatiotemporal features as STIPs, have revealed excellent performances (e.g., [114][96]), achieving 100\% accuracy on some of the common benchmark datasets. Low-level features benefit from the fact that they are generally easy to extract. However, they are unable to handle the temporal structure of the action/behavior, implying the need for a higher level analysis to construct a suitable temporal model. Mid and high-level representations can bridge this gap, providing also the spatiotemporal model (motion trajectories) associated to each descriptor, both at a feature level [113] or objects level [91][121]. Also in this case, efficient performances can be achieved, as demonstrated by Sadanand and Corso [82], who reached $98.2 \%$ on the KTH dataset and $95 \%$ on the UCF Sports dataset. Enriching the descriptors with contextual information 
TABLE V: Comparison of social interaction modeling approaches (Key. SFM: Social Force Model; BoW: Bag of Words; GA: Genetic Algorithm; SVM: Support Vector Machine; EM: Expectation Maximization; CRF: Conditional Random Field; STIP: Space Time Interest Points).

\begin{tabular}{|c|c|c|c|c|}
\hline Ref. & Feature & Matching & Objective & Comments \\
\hline [156] & $\begin{array}{l}\text { Optical flow of parti- } \\
\text { cles }\end{array}$ & $\mathrm{SFM}+\mathrm{BoW}$ & Anomaly detection & $\begin{array}{l}\text { Far range, particles are assimilated to individuals, model regular and } \\
\text { abnormal flow }\end{array}$ \\
\hline [157] & Energy potentials & $\begin{array}{l}\text { Energy } \\
\text { minimization }\end{array}$ & $\begin{array}{l}\text { Learn pedestrian dynamics for trajectory } \\
\text { estimation }\end{array}$ & $\begin{array}{l}\text { Three energy potentials to be minimized: destination, constant veloc- } \\
\text { ity and avoidance }\end{array}$ \\
\hline [158] & Energy potentials & GAs. & $\begin{array}{l}\text { Improve multi-target tracking exploiting } \\
\text { social behavioral models }\end{array}$ & Energy potentials for position, speed and direction of motion \\
\hline [160] & $\begin{array}{l}\text { Potentials for distance } \\
\text { and velocity }\end{array}$ & SVM + Proxemics & $\begin{array}{l}\text { Classification of two-persons interac- } \\
\text { tions: intentional, normal, abnormal }\end{array}$ & Analysis carried out with a temporal sliding window \\
\hline [161] & Distance & $\mathrm{MRF}+$ Proxemics & Improve visual tracking & Joint analysis of social space, visual attention and personality traits \\
\hline [162] & Distance & $\begin{array}{l}\text { Clustering + EM + } \\
\text { Proxemics }\end{array}$ & $\begin{array}{l}\text { Infer social relations from interpersonal } \\
\text { distances }\end{array}$ & $\begin{array}{l}\text { Detection of F-Formations. Social distances used to infer roles of } \\
\text { people in the party }\end{array}$ \\
\hline [163] & $\begin{array}{l}\text { Visual Codebooks on } \\
\text { motion trajectories }\end{array}$ & SVM & $\begin{array}{l}\text { Recognition of self, pair, and group- } \\
\text { causality }\end{array}$ & $\begin{array}{l}\text { A dataset is also proposed. Activities include walking and running } \\
\text { together, gathering, standing, fighting }\end{array}$ \\
\hline [164] & Energy potentials & $\mathrm{CRF}$ & $\begin{array}{l}\text { Infer social interactions to improve } \\
\text { tracking in groups }\end{array}$ & $\begin{array}{l}\text { Third-order graphical model to jointly estimate trajectories and group } \\
\text { memberships over a time window }\end{array}$ \\
\hline [165] & $\begin{array}{l}\text { Energy potentials on } \\
\text { STIPs }\end{array}$ & $\mathrm{BoW}+\mathrm{SVM}$ & Detection of abnormal events & $\begin{array}{l}\text { Human detection and segmentation are not necessary. Events modeled } \\
\text { through STIPs }\end{array}$ \\
\hline
\end{tabular}

can also provide significant improvements in the classification performances. This can be achieved by complementing the features with semantics [119], defining (or learning) a description of the video in terms of causal relationships, as it is common, for example, in sport events. These approaches are particularly efficient when observing long-term actions, in which atomic elements can be combined together to compose a more complex and structured behaviors.

Although the appropriateness of the feature representation is a key element to create a correct behavior model, the choice of the classifier may strongly influence the quality of the results. Deterministic classifiers (such as neural networks, SVM), often combined with some efficient representation of the input data as bag-of-words or dimensionality reduction, are commonly used. However, a deterministic model is strongly dependent on the set of training data. For this reason, deterministic models are well suited to detect behaviors that show a certain level of regularity and for which the salient details that characterize them are persistent across the samples of the dataset. A different category of methods model behaviors as stochastic processes. A stochastic, or probabilistic process assumes that the variables to be analyzed behave as probability distributions, instead of single values, thus incorporating in the model a certain degree of randomness. Some of the most widely used include Markovian models and their variations (e.g., HMM, MRF), as well as CRF and stochastic contextfree grammars (SCFG). These solutions often match very well with the nature of behaviors that tend to evolve or change, especially when observed over long temporal intervals, due to the high variability with which humans accomplish the same tasks over time.

Graphical models [175] have demonstrated their robustness in dealing with noise both in data acquisition and behavior interpretation [176]. Examples include action [177][80][93] and interaction analysis [178][164].

The problem of reconstructing the motion of a $3 \mathrm{D}$ articulated body from $2 \mathrm{D}$ point correspondences from a single camera for behavior understanding has been gaining increased attention [179], [180]. However, methods still require capturing the object at a high resolution and proximity to the camera. New opportunities are offered by devices such as RGBDepth (RGB-D) sensors (e.g. Asus Xtion [181] and Microsoft Kinect [182]) that simplify tasks at close range for human behavior analysis [41][134][135]. Another natural extension is the use of multiple cameras [74] [139] [140]. Moreover, in order to increase the performance achieved by pure vision systems in real scenarios, multi-modal sensing should be considered [183], [184]. Examples include the integration of 2D laser scanners with cameras [183] or cameras and microphones [185].

Datasets and their field of applicability have been discussed throughout the survey and are summarized in Tables II, IV, and VI. Each dataset generally comprises a specific facet of human behavior understanding. Annotated datasets that combine detection, activity and interaction analysis are still missing and would greatly facilitate progress in this field whose building components have been traditionally analyzed separately. Moreover, especially in the area of action and interaction recognition, datasets are generally small, making it difficult to use a relevant number of examples for learning and testing. As human analysis datasets grow larger and more complex, it becomes harder to perform accurate annotation, which is paramount for training. For this reason, crowdsourcing annotation of datasets is growing in popularity [186]. Interesting quality assessment for crowdsourced object annotations have been proposed [187], illustrating the need for large and reliably annotated datasets to help increasing the performance of algorithms.

\section{REFERENCES}

[1] T. Xiang and S. Gong, "Video behavior profiling for anomaly detection," IEEE Transactions on Pattern Analysis and Machine Intelligence, vol. 30, no. 5, pp. 893-908, 2008.

[2] M. Pantic, A. Pentland, A. Nijholt, and T. Huang, "Human computing and machine understanding of human behavior: A survey," Artifical Intelligence for Human Computing, pp. 47-71, 2007. 
TABLE VI: Datasets for action and interaction recognition. (Key. A: Annotation; Y: yes; N: no; P: partial)

\begin{tabular}{|c|c|c|c|c|}
\hline Ref. & Name & Features & $\mathbf{A}$ & Comments \\
\hline [78] & KTH dataset & $\begin{array}{l}2391 \text { sequences at } 25 \mathrm{fps} \text {, downsampled to } \\
160 \times 120 \text { pixels. }\end{array}$ & $\mathrm{Y}$ & $\begin{array}{l}25 \text { subjects repeating six actions (walking, jogging, running, boxing, hand } \\
\text { waving and hand clapping) in four scenarios }\end{array}$ \\
\hline [136] & Weizmann dataset & 90 sequences, of resolution $80 \times 144$ at $50 \mathrm{fps}$ & $\mathrm{Y}$ & $\begin{array}{l}9 \text { people performing } 10 \text { natural actions: run, walk, skip, jump-in-place, } \\
\text { jumping-jack, jump-forward-on-two-legs, wave-one-hand, wave-two-hands, } \\
\text { bend, gallopsideways. }\end{array}$ \\
\hline [139] & INRIA Xmas & $\begin{array}{l}429 \text { sequences of resolution } 390 \times 291 \text { at } 25 \\
\text { fps }\end{array}$ & $\mathrm{Y}$ & $\begin{array}{l}5 \text {-view recording of } 13 \text { daily-live motions performed each } 3 \text { times by } 11 \text { actors. } \\
\text { The actors choose freely position and orientation }\end{array}$ \\
\hline [140] & MuHAVi & 238 sequences of resolution $704 \times 576$ & $\mathrm{Y}$ & 8-views recording of 17 action classes performed by 14 actors \\
\hline [111] & UCF YouTube database & $\begin{array}{l}1100 \text { sequences of resolution } 320 \times 240 \text { at } 30 \\
\text { fps }\end{array}$ & $\mathrm{Y}$ & $\begin{array}{l}\text { Non-staged, from YouTube, activities include cycling, diving, jumping and } \\
\text { horse riding }\end{array}$ \\
\hline [138] & UCF50 & 50 actions & $\mathrm{Y}$ & Extension of UCF Youtube database \\
\hline $\begin{array}{l}{[137],} \\
{[112]}\end{array}$ & $\begin{array}{l}\text { Hollywood Human Ac- } \\
\text { tion datasets }\end{array}$ & Samples from 12 movies, at 25 fps. & $\mathrm{Y}$ & Non-staged human actions from feature films, sitcoms and news segments \\
\hline [141] & 3DPES & $\begin{array}{l}\text { Recording over several days of more than } 200 \\
\text { people }\end{array}$ & $\mathrm{Y}$ & $\begin{array}{l}\text { Multi-camera videos with non-overlapped field of view, designed for re- } \\
\text { identification. }\end{array}$ \\
\hline [142] & MIT trajectory dataset & Contains more than 40000 trajectories & - & Videos recorded from a camera monitoring a parking lot. \\
\hline [143] & EIFPD & about 92000 trajectories, 8 millions of targets & $\mathrm{N}$ & Data recorded over months of working days. \\
\hline [170] & BEHAVE & 10 types of interactions & $\mathrm{P}$ & $\begin{array}{l}\text { Types of interactions: in group, approach, walk together, split, ignore, follow- } \\
\text { ing, chase, fight, run together, and meet }\end{array}$ \\
\hline [116] & UT Interaction & 20 videos, 6 types of interactions & $\mathrm{Y}$ & $\begin{array}{l}\text { Small dataset for simple human interactions (hug, hand-shake, push, kick, } \\
\text { punch, point) }\end{array}$ \\
\hline [172] & MOCAP & about 110 videos & $\mathrm{Y}$ & 3D dataset including two-person interactions \\
\hline [171] & $\begin{array}{l}\text { TV Human Interactions } \\
\text { Dataset }\end{array}$ & 300 videos, 4 interactions & $\mathrm{Y}$ & Short clips from TV-Shows including kiss, hug, high-five, hand-shake \\
\hline [152] & RGBD-HuDaAct & $\begin{array}{l}\text { RGB-D data in } 1189 \text { videos ( } 46 \text { hours) at } 30 \\
\text { fps and resolution } 640 \times 480\end{array}$ & $\mathrm{Y}$ & $\begin{array}{l}\text { One single subject is present in each action. Each sequence spans about } 30 \text { to } \\
50 \text { seconds }\end{array}$ \\
\hline [134] & CAD-60 & $\begin{array}{l}\text { RGB-D data in } 60 \text { videos at } 30 \text { fps and resolu- } \\
\text { tion } 640 \times 480\end{array}$ & $\mathrm{Y}$ & $\begin{array}{l}3 \text { to } 4 \text { common household activities identified in each location about } 45 \\
\text { seconds of data for each activity and each person }\end{array}$ \\
\hline [169] & CAD- 120 & $\begin{array}{l}\text { RGB-D data in } 120 \text { activities, at } 30 \mathrm{fps} \text { and } \\
\text { resolution } 640 \times 480\end{array}$ & $\mathrm{Y}$ & tracked human skeleton joints and tracked object bounding boxes are annotated \\
\hline [174] & LIRIS & $\begin{array}{l}\text { RGB-D data in } 828 \text { actions at } 25 \text { fps and } 640 \times \\
480 \text { (RGB-D) }+720 \times 576 \text { (RGB) }\end{array}$ & $\mathrm{Y}$ & $\begin{array}{l}\text { In addition to RGB-D standard video of the same scenes, recorded with a } \\
\text { consumer camcorder, for comparisons between higher quality RGB and RGB- } \\
\text { D techniques }\end{array}$ \\
\hline
\end{tabular}

[3] A. Shirai, E. Geslin, and S. Richir, "Wiimedia: motion analysis methods and applications using a consumer video game controller," in $A C M$ SIGGRAPH Symposium on Video Games. ACM, 2007, pp. 133-140.

[4] L. Yin, X. Wei, Y. Sun, J. Wang, and M. Rosato, "A 3d facial expression database for facial behavior research," in International Conference on Automatic Face and Gesture Recognition. IEEE, 2006, pp. 211-216.

[5] C. Kidd, R. Orr, G. Abowd, C. Atkeson, I. Essa, B. MacIntyre, E. Mynatt, T. Starner, and W. Newstetter, "The aware home: A living laboratory for ubiquitous computing research," Cooperative buildings. Integrating information, organizations, and architecture, pp. 191-198, 1999.

[6] Z. Niu, X. Gao, and Q. Tian, "Tactic analysis based on real-world ball trajectory in soccer video," Pattern Recognition, 2011.

[7] C. Snoek, V. Nedovic, M. van Liempt, R. van Balen, F. Yan, M. Tahir, K. Mikolajczyk, J. Kittler, M. de Rijke, J. Geusebroek et al., "The mediamill trecvid 2008 semantic video search engine," 2009.

[8] Z. Zhang and D. Tao, "Slow feature analysis for human action recognition," Pattern Analysis and Machine Intelligence, IEEE Transactions on, vol. 34, no. 3, pp. 436-450, 2012.

[9] A. Yao, J. Gall, and L. Van Gool, "Coupled action recognition and pose estimation from multiple views," International journal of computer vision, pp. 1-22, 2012.

[10] S. Ba and J. Odobez, "Multiperson visual focus of attention from head pose and meeting contextual cues," Pattern Analysis and Machine Intelligence, IEEE Transactions on, vol. 33, no. 1, pp. 101-116, 2011.

[11] M. Enzweiler and D. M. Gavrila, "Monocular pedestrian detecion: Survey and experiments," IEEE Transactions on Pattern Analysis and Machine Intelligence, vol. 31, no. 12, pp. 2179-2195, December 2009.

[12] D. Gerónimo, A. M. López, A. D. Sappa, and T. Graf, "Survey of pedestrian detection for advanced driver assistance systems," IEEE Transactions on Pattern Analysis and Machine Intelligence, vol. 32, no. 7, pp. 1239-1258, July 2010.

[13] P. Dollár, C. Wojek, B. Schiele, and P. Perona, "Pedestrian detection:

A benchmark," in International Conference on Computer Vision and Pattern Recognition. IEEE, 2009, pp. 304-311.

[14] _ - "Pedestrian detection: An evaluation of the state of the art," IEEE Transactions on Pattern Analysis and Machine Intelligence, no. 4, pp. 743-761, 2012.

[15] B. Morris and M. Trivedi, "A Survey of Vision-Based Trajectory Learning and Analysis for Surveillance," IEEE Transactions on Circuits and Systems for Video Technology, vol. 18, no. 8, pp. 1114-1127, 2008.

[16] T. Moeslund, A. Hilton, and V. Kruger, "A survey of advances in vision-based human motion capture and analysis," Computer vision and image understanding, vol. 104, no. 2-3, pp. 90-126, 2006.

[17] R. Poppe, "A survey on vision-based human action recognition," Image and Vision Computing, vol. 28, no. 6, pp. 976-990, 2010.

[18] J. Aggarwal and M. Ryoo, "Human activity analysis: A review," $A C M$ Computing Surveys (CSUR), vol. 43, no. 3, p. 16, 2011.

[19] Y. Wu and T. Huang, "Vision-based gesture recognition: A review," Gesture-based communication in human-computer interaction, pp. 103-115, 1999.

[20] P. Turaga, R. Chellappa, V. Subrahmanian, and O. Udrea, "Machine recognition of human activities: A survey," IEEE Transactions on Circuits and Systems for Video Technology, vol. 18, no. 11, pp. 1473$1488,2008$.

[21] A. Maslow, R. Frager, and J. Fadiman, Motivation and personality. Harper \& Row New York, 1970, vol. 2.

[22] J. Baird and D. Baldwin, "Making sense of human behavior: Action parsing and intentional inference," Intentions and intentionality: Foundations of social cognition, pp. 193-206, 2001.

[23] J. Thibaut and H. Kelley, "The social psychology of groups." 1959.

[24] W. Doise and A. Palmonari, Social interaction in individual development. Cambridge Univ Pr, 1984, vol. 3.

[25] N. Dalal, B. Triggs, I. Rhone-Alps, and F. Montbonnot, "Histogram of oriented gradients for human detection," in IEEE International 
Conference on Computer Vision and Pattern Recognition, vol. 1, 2005, pp. 886-893.

[26] L. Pishchulin, A. Jain, C. Wojek, M. Andriluka, T. Thormahlen, and B. Schiele, "Learning people detection models from few training samples," in IEEE Conference on Computer Vision and Pattern Recognition. IEEE, June 2011, pp. 1473-1480.

[27] Z. Lin and L. Davis, "A pose-invariant descriptor for human detection and segmentation," in European Conference on Computer Vision, 2008, pp. $423-436$

[28] B. Leibe, E. Seemann, and B. Schiele, "Pedestrian detection in crowded scenes," in IEEE International Conference on Computer Vision and Pattern Recognition, vol. 1, 2005, pp. 878-885.

[29] M. Andriluka, S. Roth, and B. Schiele, "Pictorial structures revisited: People detection and articulated pose estimation," in IEEE Conference on Computer Vision and Pattern Recognition. IEEE, 2009, pp. 1014 1021.

[30] S. Johnsen and A. Tews, "Real-time object tracking and classification using a static camera," in IEEE International Conference on Robotics and Automation - Workshop on People Detection and Tracking, May 2009

[31] Q. Meng, B. Li, and H. Holstein, "Recognition of human periodic movements from unstructured information using a motion-based frequency domain approach," Image and Vision Computing, vol. 24, no. 8, pp. 795-809, August 2006.

[32] Y. Liu, X. Chen, H. Yao, X. Cui, C. Liu, and W. Gao, "Contour-motion feature (cmf): a space-time approach for robust pedestrian detection," Pattern Recognition Letters, vol. 30, no. 2, pp. 148-156, 2009.

[33] Y. Chen, Q. Wu, and X. He, "Motion based pedestrian recognition," in International Congress on Image and Signal Processing, 2008, pp. 376-380.

[34] Y. Ran, I. Weiss, Q. Zheng, and L. S. Davis, "Pedestrian detection via periodic motion analysis," International Journal of Computer Vision, vol. 71, no. 2, pp. 143-160, February 2007.

[35] S. Walk, N. Majer, K. Schindler, and B. Schiele, "New features and insights for pedestrian detection," in IEEE International Conference on Computer Vision and Pattern Recognition, 2010, pp. 1030-1037.

[36] B. Babenko, P. Dollár, Z. Tu, S. Belongie et al., "Simultaneous learning and alignment: Multi-instance and multi-pose learning," 2008.

[37] F. Perbet, A. Maki, and B. Stenger, "Correlated probabilistic trajectories for pedestrian motion detection," in IEEE International Conference on Computer Vision. IEEE, 2009, pp. 1647-1654.

[38] P. V. K. Borges, "Blob motion statistics for pedestrian detection," in International Conference on Digital Image Computing Techniques and Applications. IEEE, 2011, pp. 442-447.

[39] J. Ge, Y. Luo, and G. Tei, "Real-time pedestrian detection and tracking at nighttime for driver-assistance systems," IEEE Transactions on Intelligent Transportation Systems, vol. 10, no. 2, pp. 283-298, 2009.

[40] L. Spinello and K. O. Arras, "People detection in rgb-d data," in Intelligent Robots and Systems (IROS), 2011 IEEE/RSJ International Conference on. IEEE, 2011, pp. 3838-3843.

[41] L. Xia, C. Chen, and J. Aggarwal, "Human detection using depth information by kinect," in Workshop on Human Activity Understanding from 3D Data in conjunction with IEEE Conference on Computer Vision and Pattern Recognition, 2011, pp. 15-22.

[42] D. Gavrila and S. Munder, "Multi-cue pedestrian detection and tracking from a moving vehicle," International Journal of Computer Vision, vol. 73, no. 1, pp. 41-59, 2007.

[43] A. Howard, L. Matthies, A. Huertas, M. Bajracharya, and A. Rankin, "Detecting pedestrians with stereo vision: safe operation of autonomous ground vehicles in dynamic environments," in Proceedings of the 13th International Symposium of Robotics Research, 2007, pp. 26-29.

[44] P. Viola and M. Jones, "Rapid object detection using a boosted cascade of simple features," in IEEE Conference on Computer Vision and Pattern Recognition, vol. 1. IEEE, 2001, pp. I-511.

[45] S. Paisitkriangkrai, C. Shen, and J. Zhang, "Fast pedestrian detection using a cascade of boosted covariance features," Circuits and Systems for Video Technology, IEEE Transactions on, vol. 18, no. 8, pp. 11401151, 2008.

[46] P. Felzenszwalb, R. Girshick, D. McAllester, and D. Ramanan, "Object detection with discriminatively trained part-based models," IEEE Transactions on Pattern Analysis and Machine Intelligence, pp. 1627$1645,2009$.

[47] M. Enzweiler and D. M. Gavrila, "Combination of feature extraction method for svm pedestrian detection," IEEE Transactions on Intelligent Transportation Systems, vol. 8, no. 2, pp. 292-307, June 2007.
[48] P. Viola, M. Jones, and D. Snow, "Detecting pedestrians using patterns of motion and appearance," International Journal of Computer Vision, vol. 63, no. 2, pp. 153-161, 2005.

[49] INRIA. (2012) Person dataset. [Online]. Available: http://pascal. inrialpes.fr/data/human/

[50] T. Bouwmans, F. E. Baf, and B. Vachon, Statistical Background Modeling for Foreground Detection: A Survey. World Scientific Publishing, 2010, vol. 4, ch. 3, pp. 181-199.

[51] B. Wu and R. Nevatia, "Detection and segmentation of multiple, partially occluded objects by grouping, merging, assigning part detection responses," International Journal of Computer Vision, vol. 82, no. 2, pp. 185-204, April 2009.

[52] P. Borges, "Pedestrian detection based on blob motion statistics," IEEE Transactions on Circuits and Systems for Video Technology, vol. 23, no. 2, pp. 224-235, 2013.

[53] J. J. Little and J. E. Boyd, "Recognizing people by their gait: The shape of motion," Videre Journal of Computer Vision Research, vol. 1, no. 2, pp. 1-32, 1998.

[54] P. S. Tsai, M. Shah, K. Keiter, and T. Kasparis, "Cyclic motion detection for motion based recognition," Pattern Recognition, vol. 27 , no. 12, pp. 1591-1603, December 1994.

[55] R. Cutler and L. Davis, "Robust real-time periodic motion detection, analysis, and applications," IEEE Transactions on Pattern Analysis and Machine Intelligence, vol. 22, no. 8, pp. 781-796, August 2000.

[56] J. E. Boyd, "Synchronization of oscillations for machine perception of gaits," Computer Vision and Image Understanding, vol. 96, pp. 35-59, 2004.

[57] B. G. Quinn and E. J. Hannan, The Estimation and Tracking of Frequency. Cambridge University Press, 2001, pp. 143-162.

[58] D. G. Manolakis, V. K. Ingle, and S. M. Kogon, Statistical and Adaptive Signal Processing. McGraw-Hill, 2000.

[59] A. Efros, A. Berg, G. Mori, and J. Malik, "Recognizing action at a distance," in IEEE International Conference on Computer Vision. IEEE, 2003, pp. 726-733.

[60] X. Wang, X. Ma, and W. Grimson, "Unsupervised activity perception in crowded and complicated scenes using hierarchical bayesian models," IEEE Transactions on Pattern Analysis and Machine Intelligence, pp. 539-555, March 2009.

[61] M. Wang and X. Wang, "Automatic adaptation of a generic pedestrian detector to a specific traffic scene," in IEEE Conference on Computer Vision and Pattern Recognition. IEEE, June 2011, pp. 3401-3408.

[62] J. Rosell, G. Andreu, A. Rodas, V. Atienza, and J. Valiente, "Feature sets for people and luggage recognition in airport surveillance under real-time constraints," VISIGRAPP08, pp. 662-665, 2008.

[63] X. Liu, L. Lin, S. Yan, H. Jin, and W. Tao, "Integrating spatiotemporal context with multiview representation for object recognition in visual surveillance," Circuits and Systems for Video Technology, IEEE Transactions on, vol. 21, no. 4, pp. 393-407, 2011.

[64] T. Elguebaly and N. Bouguila, "A nonparametric bayesian approach for enhanced pedestrian detection and foreground segmentation," in IEEE Conference on Computer Vision and Pattern Recognition. IEEE, June 2011, pp. 21-26.

[65] C. Dai, Y. Zheng, and X. Li, "Pedestrian detection and tracking in infrared imagery using shape and appearance," Computer Vision and Image Understanding, vol. 106, no. 2-3, pp. 288-299, 2007.

[66] N. Dalal, "Finding people in images and videos," PHDs thesis, Institut National Polytechnique de Grenoble, 2006.

[67] Y. Freund and R. Schapire, "A desicion-theoretic generalization of online learning and an application to boosting," in Computational learning theory. Springer, 1995, pp. 23-37.

[68] M. Jones and D. Snow, "Pedestrian detection using boosted features over many frames," in International Conference on Pattern Recognition. IEEE, 2008, pp. 1-4.

[69] I. Bouchrika, J. Carter, M. Nixon, R. Morzinger, and G. Thallinger, "Using gait features for improving walking people detection," in IEEE International Conference on Pattern Recognition. IEEE, 2010, pp. 3097-3100.

[70] W. Ge, R. Collins, and R. Ruback, "Vision-based analysis of small groups in pedestrian crowds," IEEE Transactions on Pattern Analysis and Machine Intelligence, vol. 34, no. 5, pp. 1003-1016, 2012.

[71] MIT. (2000) Cbcl pedestrian database. [Online]. Available: http: //cbcl.mit.edu/cbcl/software-datasets/PedestrianData.html

[72] B. Wu and R. Nevatia, "Cluster boosted tree classifier for multi-view, multi-pose object detection," in IEEE International Conference on Computer Vision. IEEE, 2007, pp. 1-8. 
[73] — "Detection of multiple, partially occluded humans in a single image by bayesian combination of edgelet part detectors," in IEEE International Conference on Computer Vision, vol. 1. IEEE, 2005, pp. 90-97.

[74] A. Ess, B. Leibe, and L. Van Gool, "Depth and appearance for mobile scene analysis," in IEEE International Conference on Computer Vision. IEEE, 2007, pp. 1-8.

[75] PETS. (2005) Computational vision group - university of reading, uk. [Online]. Available: http://www.cvg.cs.rdg.ac.uk

[76] CAVIAR, "Ec funded caviar project/ist 200137540 dataset," 2001 [Online]. Available: http://homepages.inf.ed.ac.uk/rbf/CAVIAR/

[77] I. Laptev, "On space-time interest points," International Journal of Computer Vision, vol. 64, no. 2, pp. 107-123, 2005.

[78] C. Schuldt, I. Laptev, and B. Caputo, "Recognizing human actions: A local svm approach," in International Conference on Pattern Recognition, vol. 3. IEEE, 2004, pp. 32-36.

[79] J. Yamato, J. Ohya, and K. Ishii, "Recognizing human action in timesequential images using hidden markov model," in IEEE Conference on Computer Vision and Pattern Recognition. IEEE, 1992, pp. 379-385.

[80] W. Choi, K. Shahid, and S. Savarese, "Learning context for collective activity recognition," in Computer Vision and Pattern Recognition (CVPR), 2011 IEEE Conference on. IEEE, 2011, pp. 3273-3280.

[81] L. Lin, H. Gong, L. Li, and L. Wang, "Semantic event representation and recognition using syntactic attribute graph grammar," Pattern Recognition Letters, vol. 30, no. 2, pp. 180-186, 2009.

[82] S. Sadanand and J. Corso, "Action bank: A high-level representation of activity in video," in Computer Vision and Pattern Recognition (CVPR), 2012 IEEE Conference on. IEEE, 2012, pp. 1234-1241.

[83] A. Bobick and J. Davis, "The recognition of human movement using temporal templates," IEEE Transactions on Pattern Analysis and Machine Intelligence, vol. 23, no. 3, pp. 257-267, 2002.

[84] V. Kellokumpu, M. Pietikäinen, and J. Heikkilä, "Human activity recognition using sequences of postures," in Proceedings of the IAPR Conference on Machine Vision Applications, 2005, pp. 570-573.

[85] W. Li, Z. Zhang, and Z. Liu, "Expandable data-driven graphical modeling of human actions based on salient postures," IEEE Transactions on Circuits and Systems for Video Technology, vol. 18, no. 11, pp. 1499-1510, 2008.

[86] D. Chen, S. Shih, and H. Liao, "Human Action Recognition Using 2D Spatio-Temporal Templates," in IEEE International Conference on Multimedia and Expo. IEEE, 2007, pp. 667-670.

[87] F. Nater, H. Grabner, and L. Van Gool, "Exploiting simple hierarchies for unsupervised human behavior analysis," in IEEE Conference on Computer Vision and Pattern Recognition. IEEE, 2010, pp. 2014 2021

[88] W. Li, Z. Zhang, and Z. Liu, "Action recognition based on a bag of 3D points," in IEEE Conference on Computer Vision and Pattern Recognition. IEEE, 2010, pp. 9-14.

[89] O. Concha, D. Xu, R. Yi, Z. Moghaddam, and M. Piccardi, "Hmmmio: an enhanced hidden markov model for action recognition," in IEEE Conference on Computer Vision and Pattern Recognition. IEEE, 2011, pp. 62-69.

[90] C. Sun, I. Junejo, and H. Foroosh, "Action recognition using rank-1 approximation of joint self-similarity volume," in IEEE International Conference on Computer Vision. IEEE, 2011, pp. 1007-1012.

[91] Z. Zhang, T. Tan, and K. Huang, "An extended grammar system for learning and recognizing complex visual events," IEEE Transactions on Pattern Analysis and Machine Intelligence, vol. 33, no. 2, February 2011.

[92] M. Raptis, I. Kokkinos, and S. Soatto, "Discovering discriminative action parts from mid-level video representations," in Computer Vision and Pattern Recognition (CVPR), 2012 IEEE Conference on. IEEE, 2012, pp. 1242-1249.

[93] M. Hoai, Z. Lan, and F. De la Torre, "Joint segmentation and classification of human actions in video," in Computer Vision and Pattern Recognition (CVPR), 2011 IEEE Conference on. IEEE, 2011, pp. 3265-3272.

[94] S. Calderara, U. Heinemann, A. Prati, R. Cucchiara, and R. Naftali, "Detecting anomalies in people's trajectories using spectral graph analysis," Computer Vision and Image Understanding, 2011.

[95] H. Wang, A. Klaser, C. Schmid, and C.-L. Liu, "Action recognition by dense trajectories," in IEEE Conference on Computer Vision and Pattern Recognition. IEEE, 2011, pp. 3169-3176.

[96] A. Castrodad and G. Sapiro, "Sparse modeling of human actions from motion imagery," International journal of computer vision, vol. 100, no. 1 , pp. 1-15, 2012.
[97] S. Wu, O. Oreifej, and M. Shah, "Action recognition in videos acquired by a moving camera using motion decomposition of lagrangian particle trajectories," in IEEE International Conference on Computer Vision. IEEE, 2011, pp. 1419-1426.

[98] P. Dollar, V. Rabaud, G. Cottrell, and S. Belongie, "Behavior recognition via sparse spatio-temporal features," in Visual Surveillance and Performance Evaluation of Tracking and Surveillance, 2005. 2nd Joint IEEE International Workshop on. IEEE, 2005, pp. 65-72.

[99] J. Niebles, H. Wang, and L. Fei-Fei, "Unsupervised learning of human action categories using spatial-temporal words," International Journal of Computer Vision, vol. 79, no. 3, pp. 299-318, 2008.

[100] H. Jhuang, T. Serre, L. Wolf, and T. Poggio, "A biologically inspired system for action recognition," in Computer Vision, 2007. ICCV 2007. IEEE 11th International Conference on. Ieee, 2007, pp. 1-8.

[101] J. Liu and M. Shah, "Learning human actions via information maximization," in IEEE Conference on Computer Vision and Pattern Recognition. IEEE, 2008, pp. 1-8.

[102] M. Ryoo and J. Aggarwal, "Spatio-temporal relationship match: Video structure comparison for recognition of complex human activities," in IEEE International Conference on Computer Vision. IEEE, 2009, pp. $1593-1600$.

[103] I. Laptev and T. Lindeberg, "Space-time interest points," in IEEE International Conference on Computer Vision. IEEE, 2003, pp. 432439.

[104] C. Harris and M. Stephens, "A combined corner and edge detector," in Alvey Vision Conference, vol. 15. Manchester, UK, 1988, p. 50.

[105] M. Bregonzio, S. Gong, and T. Xiang, "Recognising action as clouds of space-time interest points," in IEEE Conference on Computer Vision and Pattern Recognition. IEEE, 2009, pp. 1948-1955.

[106] M. Ullah, S. Parizi, and I. Laptev, "Improving bag-of-features action recognition with non-local cues," in British Machine Vision Conference, vol. 2, 2010, p. 7.

[107] D. Han, L. Bo, and C. Sminchisescu, "Selection and context for action recognition," in IEEE International Conference on Computer Vision. IEEE, 2009, pp. 1933-1940.

[108] J. Sun, X. Wu, S. Yan, L. Cheong, T. Chua, and J. Li, "Hierarchical spatio-temporal context modeling for action recognition," in IEEE Conference on Computer Vision and Pattern Recognition. Ieee, 2009, pp. 2004-2011.

[109] B. Chakraborty, M. Holte, T. Moeslund, J. Gonzalez, and F. Roca, "A selective spatio-temporal interest point detector for human action recognition in complex scenes," in IEEE International Conference on Computer Vision. IEEE, 2011, pp. 1776-1783.

[110] H. Wang, A. Klaser, C. Schmid, and C.-L. Liu, "Dense trajectories and motion boundary descriptors for action recognition," International Journal of Computer Vision, vol. 103, no. 1, pp. 60-79, 2013.

[111] J. Liu, J. Luo, and M. Shah, "Recognizing realistic actions from videos in the wild," in IEEE Conference on Computer Vision and Pattern Recognition. IEEE, 2009, pp. 1996-2003.

[112] M. Marszalek, I. Laptev, and C. Schmid, "Actions in context," in IEEE Conference on Computer Vision and Pattern Recognition. IEEE, 2009, pp. 2929-2936.

[113] M. D. Rodriguez, J. Ahmed, and M. Shah, "Action mach: a spatiotemporal maximum average correlation height filter for action recognition," in IEEE Conference on Computer Vision and Pattern Recognition. IEEE, 2008, pp. 1-8.

[114] C. Chen and J. Aggarwal, "Modeling human activities as speech," in IEEE Conference on Computer Vision and Pattern Recognition. IEEE, 2011, pp. 3425-3432.

[115] M. Ryoo, "Human activity prediction: Early recognition of ongoing activities from streaming videos," in IEEE International Conference on Computer Vision. IEEE, 2011, pp. 1036-1043.

[116] M. S. Ryoo and J. K. Aggarwal. (2010) UT-Interaction Dataset, ICPR contest on Semantic Description of Human Activities (SDHA) [Online]. Available: http://cvrc.ece.utexas.edu/SDHA2010/Human $\searrow_{-}$ Interaction.html

[117] W. Choi, K. Shahid, and S. Savarese, "What are they doing?: Collective activity classification using spatio-temporal relationship among people," in IEEE International Conference on Computer Vision Workshops (ICCV Workshops). IEEE, 2009, pp. 1282-1289.

[118] M. Daldoss, N. Piotto, N. Conci, and F. G. B. De Natale, "Learning and matching human activities using regular expressions," in IEEE International Conference on Image Processing, 2010, pp. 4681-4684.

[119] A. Gupta, P. Srinivasan, J. Shi, and L. S. Davis, "Understanding videos, constructing plots learning a visually grounded storyline model from annotated videos," in IEEE Conference on Computer Vision and Pattern Recognition. IEEE, 2009, pp. 2012-2019. 
[120] M. Raptis and L. Sigal, "Poselet key-framing: A model for human activity recognition," in IEEE Conference on Computer Vision and Pattern Recognition, to appear. IEEE, 2013.

[121] L. Bourdev and J. Malik, "Poselets: Body part detectors trained using 3d human pose annotations," in IEEE International Conference on Computer Vision. IEEE, 2009, pp. 1365-1372.

[122] L. Li, H. Su, E. Xing, and L. Fei-Fei, "Object bank: A high-level image representation for scene classification and semantic feature sparsification," Advances in Neural Information Processing Systems, vol. 24, 2010.

[123] R. Messing, "Human activity recognition in video: extending statistical features across time, space and semantic context," Ph.D. dissertation, University of Rochester, 2011.

[124] R. Messing, C. Pal, and H. Kautz, "Activity recognition using the velocity histories of tracked keypoints," in IEEE International Conference on Computer Vision. IEEE, 2009, pp. 104-111.

[125] A. Fathi, X. Ren, and J. M. Rehg, "Learning to recognize objects in egocentric activities," in IEEE Conference on Computer Vision and Pattern Recognition. IEEE, 2011, pp. 3281-3288.

[126] M. Hu, "Visual pattern recognition by moment invariants," IRE Transactions on Information Theory, vol. 8, no. 2, pp. 179-187, 1962.

[127] J. Davis and A. Tyagi, "Minimal-latency human action recognition using reliable-inference," Image and Vision Computing, vol. 24, no. 5, pp. $455-472,2006$.

[128] L. Wang and D. Suter, "Recognizing human activities from silhouettes: Motion subspace and factorial discriminative graphical model," in IEEE Conference on Computer Vision and Pattern Recognition. IEEE, 2007, pp. $1-8$.

[129] X. Ji, H. Liu, and Y. Li, "Viewpoint insensitive actions recognition using hidden conditional random fields," Knowledge-Based and Intelligent Information and Engineering Systems, pp. 369-378, 2010.

[130] Y. Ke, R. Sukthankar, and M. Hebert, "Event detection in crowded videos," in IEEE International Conference on Computer Vision. IEEE, 2007, pp. 1-8.

[131] Y. Wang and G. Mori, "Hidden part models for human action recognition: Probabilistic vs. max-margin," IEEE Transactions on Pattern Analysis and Machine Intelligence, no. 99, pp. 1-1, 2011.

[132] H. Fujiyoshi and A. Lipton, "Real-time human motion analysis by image skeletonization," in IEEE Workshop on Applications of Computer Vision. IEEE, 1998, pp. 15-21.

[133] J. Shotton, A. Fitzgibbon, M. Cook, T. Sharp, M. Finocchio, R. Moore, A. Kipman, and A. Blake, "Real-time human pose recognition in parts from single depth images," in IEEE Conference on Computer Vision and Pattern Recognition. IEEE, 2011, pp. 1297-1304.

[134] J. Sung, C. Ponce, B. Selman, and A. Saxena, "Human activity detection from rgbd images," in AAAI workshop on Pattern, Activity and Intent Recognition (PAIR), 2011.

[135] — "Unstructured human activity detection from rgbd images," in Robotics and Automation (ICRA), 2012 IEEE International Conference on. IEEE, 2012, pp. 842-849.

[136] M. Blank, L. Gorelick, E. Shechtman, M. Irani, and R. Basri, "Actions as space-time shapes," in IEEE International Conference on Computer Vision, vol. 2. IEEE, 2005, pp. 1395-1402.

[137] I. Laptev, M. Marszalek, C. Schmid, and B. Rozenfeld, "Learning realistic human actions from movies," in IEEE Conference on Computer Vision and Pattern Recognition. IEEE, 2008, pp. 1-8.

[138] K. Reddy and M. Shah, "Recognizing 50 human action categories of web videos," Machine Vision and Applications, pp. 1-11, 2012.

[139] D. Weinland, R. Ronfard, and E. Boyer, "Free viewpoint action recognition using motion history volumes," Computer Vision and Image Understanding, vol. 104, no. 2, pp. 249-257, 2006

[140] S. Singh, S. Velastin, and H. Ragheb, "Muhavi: A multicamera human action video dataset for the evaluation of action recognition methods," in IEEE International Conference on Advanced Video and Signal Based Surveillance. IEEE Computer Society, 2010, pp. 48-55.

[141] D. Baltieri, R. Vezzani, and R. Cucchiara, "3dpes: 3d people dataset for surveillance and forensics," in Proceedings of the 1st International ACM Workshop on Multimedia access to 3D Human Objects, Scottsdale, Arizona, USA, nov 2011, pp. 59-64.

[142] E. Grimson, E. Grimson, X. Wang, G. Ng, K. Ma et al., "Trajectory analysis and semantic region modeling using a nonparametric bayesian model," 2008.

[143] EIFPD, "Edinburgh informatics forum pedestrian database." [Online]. Available: http://homepages.inf.ed.ac.uk/rbf/FORUMTRACKING/

[144] M. W. Kadous, "Temporal classification: Extending the classification paradigm to multivariate time series," Ph.D. dissertation, University of New South Wales, 2002.
[145] S. Marcel. (2000) Hand posture and gesture datasets. [Online]. Available: http://www-prima.inrialpes.fr/FGnet/data/10-Gesture/ gestures/main.html

[146] FGnet, "Two handed gesture dataset." [Online]. Available: http: //www-prima.inrialpes.fr/FGnet/data/04-TwoHand/main.html

[147] S. Marcel, O. Bernier, J. Viallet, and D. Collobert, "Hand gesture recognition using input-output hidden markov models," in IEEE International Conference on Automatic Face and Gesture Recognition. IEEE, 2000, pp. 456-461.

[148] FGnet, "Fgnet pointing'04 benchmark data." [Online]. Available: http://www-prima.inrialpes.fr/Pointing04/data-hand.html

[149] M. Holte and M. Stoerring. (2004) Pointing and command gestures under mixed illumination conditions: video sequence dataset. [Online]. Available: http://www-prima.inrialpes.fr/FGnet/data/ 03-Pointing/index.html

[150] T. Kim, S. Wong, and R. Cipolla, "Tensor canonical correlation analysis for action classification," in IEEE Conference on Computer Vision and Pattern Recognition. IEEE, 2007, pp. 1-8.

[151] ChaLearn. (2011) Gesture dataset. [Online]. Available: http:// www-prima.inrialpes.fr/FGnet/data/03-Pointing/index.html

[152] B. Ni, G. Wang, and P. Moulin, "Rgbd-hudaact: A color-depth video database for human daily activity recognition," in Consumer Depth Cameras for Computer Vision Workshop - International Conference on Computer Vision. Springer, 2013, pp. 193-208.

[153] E. Hall, The hidden dimension. Doubleday New York, 1966, vol. 6. [154] _ - The silent language. Anchor, 1973.

[155] D. Helbing and P. Molnar, "Social force model for pedestrian dynamics," Physical review E, vol. 51, no. 5, p. 4282, 1995.

[156] R. Mehran, A. Oyama, and M. Shah, "Abnormal crowd behavior detection using social force model," in IEEE Conference on Computer Vision and Pattern Recognition, june 2009, pp. 935-942.

[157] P. Scovanner and M. Tappen, "Learning pedestrian dynamics from the real world," in IEEE International Conference on Computer Vision. IEEE, 2010, pp. 381-388

[158] S. Pellegrini, A. Ess, K. Schindler, and L. van Gool, "You'll never walk alone: Modeling social behavior for multi-target tracking," in IEEE International Conference on Computer Vision, October 2009, pp. 261268.

[159] M. Taj and A. Cavallaro, "Recognizing interactions in video," Intelligent Multimedia Analysis for Security Applications, pp. 29-57, 2010.

[160] P. Rota, N. Conci, and N. Sebe, "Real time detection of social interactions in surveillance video," in Proceedings of the 3rd International Workshop on Analysis and retrieval of tracked events and motion in imagery streams, European Conference on Computer Vision, 2012.

[161] G. Zen, B. Lepri, E. Ricci, and O. Lanz, "Space speaks: towards socially and personality aware visual surveillance," in MPVA'10. ACM, 2010, pp. 37-42.

[162] M. Cristani, G. Paggetti, A. Vinciarelli, L. Bazzani, G. Menegaz, and V. Murino, "Towards computational proxemics: Inferring social relations from interpersonal distances," in SocialCom, 2011, pp. 290297.

[163] B. Ni, S. Yan, and A. Kassim, "Recognizing human group activities with localized causalities," in IEEE Conference on Computer Vision and Pattern Recognition. IEEE, 2009, pp. 1470-1477.

[164] S. Pellegrini, A. Ess, and L. Van Gool, "Improving data association by joint modeling of pedestrian trajectories and groupings," European Conference on Computer Vision, pp. 452-465, 2010.

[165] X. Cui, Q. Liu, M. Gao, and D. Metaxas, "Abnormal detection using interaction energy potentials," in IEEE Conference on Computer Vision and Pattern Recognition, 2011, pp. 3161-3167.

[166] J. Lei, X. Ren, and D. Fox, "Fine-grained kitchen activity recognition using rgb-d," in Proceedings of the 2012 ACM Conference on Ubiquitous Computing. ACM, 2012, pp. 208-211.

[167] H. Zhang and L. E. Parker, "4-dimensional local spatio-temporal features for human activity recognition," in IEEE/RSJ International Conference on Intelligent Robots and Systems. IEEE, 2011, pp. 20442049.

[168] D. M. Blei, A. Y. Ng, and M. I. Jordan, "Latent dirichlet allocation," the Journal of machine Learning research, vol. 3, pp. 993-1022, 2003.

[169] H. Swetha Koppula, R. Gupta, and A. Saxena, "Learning human activities and object affordances from rgb-d videos," 2013.

[170] BEHAVE, "The behave dataset," 2007. [Online]. Available: http: //homepages.inf.ed.ac.uk/rbf/BEHAVE

[171] A. Patron-Perez. (2010) Tv interactions dataset. [Online]. Available: http://www.robots.ox.ac.uk/ vgg/data/tv_human_ interactions/index.html 
[172] CMU, "Cmu graphics lab motion capture database," 2013. [Online]. Available: http://mocap.cs.cmu.edu/

[173] A. Patron-Perez, M. Marszalek, A. Zisserman, and I. Reid, "High five: Recognising human interactions in tv shows," 2010.

[174] C. Wolf, J. Mille, E. Lombardi, O. Celiktutan, M. Jiu, M. Baccouche, E. Dellandréa, C.-E. Bichot, C. Garcia, and B. Sankur, "The liris human activities dataset and the icpr 2012 human activities recognition and localization competition," Technical Report LIRIS RR-2012-004, Laboratoire dInformatique en Images et Systmes dInformation, INSA de Lyon, France, Tech. Rep., 2012.

[175] M. J. Wainwright and M. I. Jordan, "Graphical models, exponential families, and variational inference," Found. Trends Mach. Learn., vol. 1, no. 1-2, pp. 1-305, Jan. 2008. [Online]. Available: http://dx.doi.org/10.1561/2200000001

[176] K. Kitani, B. Ziebart, J. Bagnell, and M. Hebert, "Activity forecasting," in European Conference on Computer Vision. Springer, 2012.

[177] M. Amer, D. Xie, M. Zhao, S. Todorovic, and S. Zhu, "Cost-sensitive top-down/bottom-up inference for multiscale activity recognition," in European Conference on Computer Vision. Springer, 2012.

[178] W. Choi and S. Savarese, "A unified framework for multi-target tracking and collective activity recognition," European Conference on Computer Vision, pp. 215-230, 2012.

[179] J. Valmadre, Y. Zhu, S. Sridharan, and S. Lucey, "Efficient articulated trajectory reconstruction using dynamic programming and filters," in European Conference on Computer Vision, 2012, pp. 72-85.

[180] X. Wei and J. Chai, "Modeling 3d human poses from uncalibrated monocular images," in IEEE International Conference on Computer Vision. IEEE, 2009, pp. 1873-1880.

[181] Asus, "Xtion pro," 2012. [Online]. Available: http://www.asus.com/ Multimedia/Motion_Sensor/Xtion_PRO/

[182] Microsoft. (2013) Microsoft kinect. [Online]. Available: http: //www.xbox.com/en-GB/Kinect

[183] L. Oliveira, U. Nunes, P. Peixoto, M. Silva, and F. Moita, "Semantic fusion of laser and vision in pedestrian detection," Pattern Recognition, vol. 43 , no. 10 , pp. $3648-3659,2010$.

[184] A. Broggi, P. Cerri, S. Ghidoni, P. Grisleri, and H. Jung, "A new approach to urban pedestrian detection for automatic braking," IEEE Transactions on Intelligent Transportation Systems, vol. 10, no. 4, pp. 594-605, 2009.

[185] M. Taj and A. Cavallaro, "Interaction recognition in wide areas using audiovisual sensors," in IEEE International Conference on Image Processing, 2012.

[186] C. Vondrick, D. Ramanan, and D. Patterson, "Efficiently scaling up video annotation with crowdsourced marketplaces," European Conference on Computer Vision, pp. 610-623, 2010.

[187] S. Vittayakorn and J. Hays, "Quality assessment for crowdsourced object annotations," in British Machine Vision Conference, 2011, pp. $109-1$.

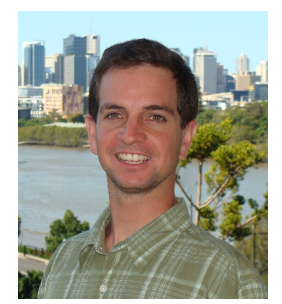

Paulo Vinicius Koerich Borges received the B.E. and M.Sc. degrees in electrical engineering from Federal University of Santa Catarina (UFSC), Brazil, in 2002 and 2004, respectively. In 2001, he was a visiting research student in the subject of image restoration at the University of Manchester, UK. In 2007 he received his Ph.D. degree from Queen Mary, University of London (QMUL). From 2007 to 2008, he was as post-doc researcher at QMUL, working on the subject of video event detection.

Since 2009, he has been working as a research scientist at the Autonomous System Laboratory at CSIRO in Brisbane, Australia. His current research topic is visual-based robot localization and pedestrian tracking. His research interests include video event detection/classification, video tracking and digital watermarking, besides general image processing and pattern recognition. Dr. Borges is also an Adjunct Lecturer with the School of Information Technology and Electrical Engineering at the University of Queensland, Australia.

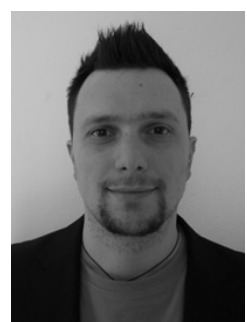

Nicola Conci (S'04-M'08) received the Ph.D in 2007 from the University of Trento, Italy. In 2007 he was visiting student at the Image Processing Lab. at University of California, Santa Barbara. In 20082009 he was post-doc researcher in the Multimedia and Vision research group at Queen Mary, University of London (UK). Since 2009 he is assistant professor at the University of Trento, Italy. His research interests are related to machine vision for behavior understanding and human computer interfaces. In 2006 he received the Best Student Paper Award at the international ACM conference Mobimedia held in Alghero (Italy).

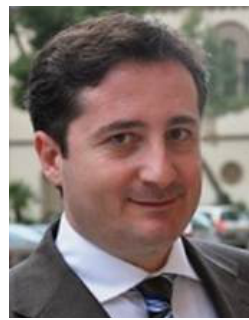

Andrea Cavallaro received the Laurea degree (summa cum laude) from the University of Trieste, Italy, in 1996, and the Ph.D. degree from the Swiss Federal Institute of Technology (EPFL), Lausanne, Switzerland, in 2002, both in electrical engineering. He served as a Research Consultant with the Image Processing Laboratory, University of Trieste, in 1996 and 1998, focusing on compression algorithms for very low bitrate video coding. From 1998 to 2003, he was a Research Assistant with the Signal Processing Laboratory, Swiss Federal Institute of Technology (EPFL), Lausanne, Switzerland. Since 2003, he has been with Queen Mary University of London, London, U.K., where he is a Professor of multimedia signal processing. He has authored more than 130 papers and published two books, Multi-Camera Networks (Elsevier, 2009) and Video Tracking (Wiley, 2011). Prof. Cavallaro was the recipient of a Research Fellowship from British Telecommunications (BT) in 2004 and 2005, three Student Paper Awards from IEEE International Conference on Acoustic, Speech, and Signal Processing in 2005, 2007, and 2009, and the Best Paper Award from the IEEE Advanced Video and Signal-based Surveillance (AVSS) in 2009. He is an elected member of the IEEE Image, Video, and Multidimensional Signal Processing Technical Committee; he served as Technical Chair for IEEE AVSS 2011, the Workshop on Image Analysis for Multimedia Interactive Services in 2010, and the European Signal Processing Conference in 2008, and as General Chair for IEEE/ACM International Conference on Distributed Smart Cameras in 2009, the British Machine Vision Conference in 2009, and the IEEE AVSS in 2007. He is an Area Editor for the IEEE Signal Processing Magazine and an Associate Editor for the IEEE TRANSACTIONS ON IMAGE PROCESSING and the IEEE TRANSACTIONS ON SIGNAL PROCESSING. 